\title{
New distances of unresolved dwarf elliptical galaxies in the vicinity of the Local Group ${ }^{\star}$
}

\author{
R. Rekola ${ }^{1}$, H. Jerjen ${ }^{2}$, and C. Flynn ${ }^{1}$ \\ 1 Tuorla Observatory, VISPA, University of Turku, Väisäläntie 20, 21500 Piikkiö, Finland \\ e-mail: rareko@utu.fi \\ 2 Research School of Astronomy and Astrophysics, The Australian National University, Mt Stromlo Observatory, Cotter Road, \\ Weston ACT 2611, Australia
}

Received 18 October 2004 / Accepted 8 March 2005

\begin{abstract}
We present Surface Brightness Fluctuation distances of nine early-type dwarf galaxies and the S0 galaxy NGC 4150 in the Local Volume based on deep $B$ - and $R$-band CCD images obtained with the $2.56 \mathrm{~m}$ Nordic Optical Telescope. Typically, six stellar fields at various galactocentric distances have been chosen for each galaxy as appropriately free of foreground stars and other contaminants, and Fourier analysed to determine the distances, which are found to lie in the range of 3 to $16 \mathrm{Mpc}$. The SBF method is thus demonstrated to efficiently measure distances from the ground with mid-aperture telescopes for galaxies for which only the tip of the red giant branch method in combination with the Hubble Space Telescope has been available until now. We obtained the following distance moduli: $28.11 \pm 0.15 \mathrm{mag}$ (or 4.2 $\pm 0.3 \mathrm{Mpc}$ ) for UGC 1703, $27.61 \pm 0.17 \mathrm{mag}$ (or $3.3 \pm 0.3 \mathrm{Mpc}$ ) for KDG 61, $29.00 \pm 0.27 \mathrm{mag}$ (or $6.3 \pm 0.8 \mathrm{Mpc}$ ) for UGCA 200, $27.74 \pm 0.18 \mathrm{mag}$ (or $3.5 \pm 0.3 \mathrm{Mpc}$ ) for UGC 5442, $30.22 \pm 0.17 \mathrm{mag}$ (or $11.1 \pm 0.9 \mathrm{Mpc}$ ) for UGC 5944, $30.79 \pm 0.11 \mathrm{mag}$ (or $14.4 \pm 0.7 \mathrm{Mpc}$ ) for NGC 4150 , $31.02 \pm 0.25 \mathrm{mag}$ (or $16.0 \pm 1.9 \mathrm{Mpc}$ ) for BTS $128,29.27 \pm 0.16 \mathrm{mag}$ (or $7.1 \pm 0.6 \mathrm{Mpc}$ ) for UGC 7639, $30.19 \pm 0.23 \mathrm{mag}$ (or $10.9 \pm 1.2 \mathrm{Mpc}$ ) for UGC 8799 with an alternative distance of $30.61 \pm 0.26 \mathrm{mag}$ (or $13.2 \pm 1.7 \mathrm{Mpc}$ ), and $29.60 \pm 0.20 \mathrm{mag}$ (or $8.3 \pm 0.8 \mathrm{Mpc}$ ) for UGC 8882 .
\end{abstract}

Key words. galaxies: dwarf - galaxies: stellar content - galaxies: structure - galaxies: distances and redshifts

\section{Introduction}

Recent imaging surveys of the Local Group neighbourhood have found and identified a large number of low surface brightness galaxy candidates, which potentially could be nearby dwarf galaxy systems (e.g. Côté et al. 1997; Karachentseva \& Karachentsev 1998; Jerjen et al. 2000a). Many are dwarf elliptical galaxies (dEs) located in galaxy groups as satellites of giant galaxies. Whether dEs are local or in the background is an important question to be answered. For example Moore et al. (1999) have numerically studied galactic and cluster halo substructures in a hierarchical universe, and found that simulations predict that the Milky Way galaxy would have many more dwarf satellites than are actually observed. This conflict is a strong motivation for catalogueing and obtaining accurate distances to dwarf galaxy population in the local universe. As a new and numerous target group in extragalactic studies dEs serve also to test the peculiar linearity and smoothness of the Hubble flow in the local volume. As such they may help to define, or at least refine, the dark energy solution to the problem (Baryshev et al. 2001).

Determining distances to galaxies of this type has been a challenge. They have only very little or no neutral hydrogen

\footnotetext{
^ Based on observations collected at the Nordic Optical Telescope.
}

gas, preventing their detection in the radio at $21 \mathrm{~cm}$ and their low surface brightness makes optical spectroscopy feasible only for the few brightest objects (Jerjen et al. 2000b). Instead, distances must be estimated from their stellar content. Taking advantage of the absence of the atmosphere, the Hubble Space Telescope (HST) is used to resolve dEs into stars and to measure the tip of the red giant branch (TRGB) magnitude (Karachentsev et al. 2000). However, this method is expensive in terms of integration time and becomes progressively difficult beyond a few Mpc due to crowding effects.

The Surface Brightness Fluctuation method can be applied to unresolved galaxies and thus offers an alternative to efficiently measure distances of $\mathrm{dEs}$ out to $10 \mathrm{Mpc}$ and beyond (e.g. Jerjen 2003). The method was originally introduced by Tonry \& Schneider (1988) to measure distances to high surface brightness elliptical galaxies. For low surface brightness dEs the method was developed (Jerjen et al. 1998, 2000b) and calibrated (Jerjen et al. 2001) only recently. It is based on the discrete sampling of the unresolved stellar population of a galaxy with the CCD detector and the resulting pixel-to-pixel variance due to the statistical noise in numbers of red giant branch (RGB) stars.

We can report here on new SBF distances for 10 nearby galaxies as part of our continuing project to map the galaxy 
Table 1. The sample of nearby early-type galaxies.

\begin{tabular}{lllcc}
\hline \hline Name & Location & Type & $\begin{array}{c}\text { RA } \\
\text { (J2000.0) }\end{array}$ & $\begin{array}{c}\text { Dec } \\
\text { (J2000.0) }\end{array}$ \\
\hline UGC 1703 & $\begin{array}{l}\text { NGC 784 } \\
\text { companion }\end{array}$ & dE & 021255.8 & +324851 \\
KDG 61 & M 81 & dE, N & 095703.1 & +683531 \\
UGCA 200 & Field? & dE, N & 100535.2 & -074444 \\
UGC 5442 & M 81 & dE, N & 100701.9 & +674939 \\
UGC 5944 & Leo I & dE/Irr & 105018.8 & +131627 \\
NGC 4150 & CVn I? & S0 & 121033.6 & +302406 \\
BTS 128 & Coma I & dE & 122120.1 & +294255 \\
UGC 7639 & CVn II & dS0? & 122953.4 & +473152 \\
UGC 8799 & Virgo I? & dE & 135319.4 & +054615 \\
UGC 8882 & M 101 & dE, N & 135714.6 & +540603 \\
\hline
\end{tabular}

groups and clouds beyond the Local Group out to $\approx 10 \mathrm{Mpc}$. We have studied nine dEs and one S0 type of galaxy in the northern hemisphere. In Table 1 we give a complete list of our galaxy sample including galaxy name, associated environment, morphological type within the extended Hubble classification system (Sandage \& Binggeli 1984), and coordinates. UGC 1703 is a field galaxy in the direction of NGC 784, KDG 61 and UGC 5442 are members of the M 81 group, UGCA 200 has been assumed to be a companion of NGC 3115 but we cannot confirm this assumption, UGC 5944 is a member of the Leo I group, NGC 4150 (the S0 galaxy) lies in the direction of the Canes Venatici I cloud (CVn I) - probably behind it, BTS 128 is a member of the Coma I group, UGC 7639 is a member of the Canes Venatici II cloud (CVn II), UGC 8882 is a member of the M101 group, and UGC 8799 may lie at the outskirts of the Virgo I cluster.

In Sect. 2, we describe the observations and data reduction. The SBF analysis is presented in Sect. 3. Individual galaxies are discussed in Sect. 4. Finally, we present the summary and draw the conclusions of this work in Sect. 5.

\section{Observations and reductions}

CCD images were obtained at the $2.56 \mathrm{~m}$ Nordic Optical Telescope on the nights of the 20 January 2002, 3-4 February 2002 and 25-27 February 2003. We used the Andalucia Faint Object Spectrograph and Camera (ALFOSC), which is equipped with a $2048 \times 2048$ Loral/Lesser CCD detector with a pixel size of $15 \mu \mathrm{m}$ and a plate scale of 0.188 arcsec, providing a field of view 6.4 arcmin on a side. The gain was set at $1 \mathrm{e}^{-} /$ADU. A series of six to ten images were taken in $B$ and $R$ passbands for each of the ten dwarf galaxies, along with bias frames, twilight flats and photometric standard star fields through the nights. The observing $\log$ is given in Table 2. The exposure time for individual science frames was $600 \mathrm{~s}$, with the exception of NGC 4150 for which we used $180 \mathrm{~s}$ in $R$ to avoid saturation of the central region of the galaxy. The seeing ranged from 0.8 to 1.6 arcsec and all six nights provided photometric
Table 2. Summary of observations.

\begin{tabular}{|c|c|c|c|c|c|}
\hline Name & Date & $\begin{array}{c}t \\
(\mathrm{~s})\end{array}$ & $\mathrm{F}$ & $A M$ & $\begin{array}{c}F W H M \\
\left({ }^{\prime \prime}\right) \\
\end{array}$ \\
\hline \multirow[t]{4}{*}{ UGC 1703} & 20 Jan. 2002 & $2 \times 600$ & $B$ & 1.08 & 1.1 \\
\hline & 20 Jan. 2002 & $6 \times 600$ & $R$ & 1.03 & 0.9 \\
\hline & 4 Feb. 2002 & $4 \times 600$ & $B$ & 1.15 & 1.0 \\
\hline & 4 Feb. 2002 & $1 \times 600$ & $R$ & 1.09 & 0.8 \\
\hline \multirow[t]{2}{*}{ UGCA 200} & 3 Feb. 2002 & $6 \times 600$ & $B$ & 2.01 & 1.2 \\
\hline & 3 Feb. 2002 & $6 \times 600$ & $R$ & 1.38 & 0.8 \\
\hline \multirow[t]{4}{*}{ UGC 5944} & 4 Feb. 2002 & $6 \times 600$ & $B$ & 1.13 & 1.0 \\
\hline & 4 Feb. 2002 & $6 \times 600$ & $R$ & 1.05 & 0.9 \\
\hline & 25 Feb. 2003 & $1 \times 600$ & $B$ & 1.04 & 0.9 \\
\hline & 26 Feb. 2003 & $1 \times 600$ & $R$ & 1.04 & 1.0 \\
\hline \multirow[t]{2}{*}{ UGC 8882} & 25 Feb. 2003 & $6 \times 600$ & $R$ & 1.16 & 0.8 \\
\hline & 26 Feb. 2003 & $5 \times 600$ & $B$ & 1.12 & 0.9 \\
\hline \multirow[t]{2}{*}{ UGC 5442} & 26 Feb. 2003 & $6 \times 600$ & $R$ & 1.38 & 0.9 \\
\hline & 26 Feb. 2003 & $5 \times 600$ & $B$ & 1.31 & 0.9 \\
\hline \multirow[t]{2}{*}{ NGC 4150} & 26 Feb. 2003 & $5 \times 600$ & $B$ & 1.03 & 0.9 \\
\hline & 26 Feb. 2003 & $10 \times 180$ & $R$ & 1.16 & 0.8 \\
\hline \multirow[t]{4}{*}{ UGC 8799} & 26 Feb. 2003 & $1 \times 600$ & $B$ & 1.22 & 1.2 \\
\hline & 26 Feb. 2003 & $3 \times 600$ & $R$ & 1.16 & 1.0 \\
\hline & 27 Feb. 2003 & $4 \times 600$ & $B$ & 1.16 & 1.1 \\
\hline & 27 Feb. 2003 & $3 \times 600$ & $R$ & 1.10 & 1.0 \\
\hline \multirow[t]{2}{*}{ KDG 61} & 27 Feb. 2003 & $5 \times 600$ & $B$ & 1.49 & 1.1 \\
\hline & 27 Feb. 2003 & $6 \times 600$ & $R$ & 1.38 & 1.1 \\
\hline \multirow[t]{2}{*}{ BTS 128} & 27 Feb. 2003 & $5 \times 600$ & $B$ & 1.13 & 1.0 \\
\hline & 27 Feb. 2003 & $6 \times 600$ & $R$ & 1.03 & 0.8 \\
\hline \multirow[t]{2}{*}{ UGC 7639} & 27 Feb. 2003 & $5 \times 600$ & $B$ & 1.09 & 1.1 \\
\hline & 27 Feb. 2003 & $6 \times 600$ & $R$ & 1.06 & 0.9 \\
\hline
\end{tabular}

conditions with the exception of the night of 4 February 2002, which was partly spectroscopic.

Image reduction was accomplished using routines within the IRAF ${ }^{1}$ programme. We removed the bias level from the images by using the bias frames and the overscan region of each image. Images were subsequently trimmed to $2000 \times 2000$ pixels to remove non-essential data from the borders. Finally, each object image was divided by the corresponding median combined masterflat. Photometric calibration was achieved using the Landolt (1992) standard star fields, which were regularly observed during each night. Thus we determined the photometric zero point (ZP), atmospheric extinction coefficient $(k)$ and colour term $(c)$ for each passband and night. Analysis revealed slight variation in extinction coefficients throughout the observation period. The mean $k$ value was calculated for each passband and the corresponding values of ZP and $c$ were reevaluated under this constraint. The results are summarised in Table 3. Images taken during the nights of $20 \mathrm{Jan}$. and

1 IRAF, Image Reduction and Analysis Facility, is distributed by the National Optical Astronomy Observatories, which is operated by the Association of Universities for Research in Astronomy, Inc., under contract with the National Science Foundation. 
Table 3. Photometric calibration coefficients.

\begin{tabular}{cccrr}
\hline \hline Date & F & ZP & $k$ & \multicolumn{1}{c}{$c$} \\
\hline 3 Feb. 2002 & $B$ & $25.40 \pm 0.03$ & $-0.22 \pm 0.02$ & $0.002 \pm 0.042$ \\
& $R$ & $25.22 \pm 0.02$ & $-0.08 \pm 0.01$ & $-0.029 \pm 0.061$ \\
25 Feb. 2003 & $B$ & $26.06 \pm 0.12$ & $-0.48 \pm 0.08$ & $0.040 \pm 0.011$ \\
& $R$ & $25.73 \pm 0.09$ & $-0.31 \pm 0.06$ & $-0.031 \pm 0.019$ \\
26 Feb. 2003 & $B$ & $25.77 \pm 0.04$ & $-0.26 \pm 0.02$ & $0.042 \pm 0.013$ \\
& $R$ & $25.47 \pm 0.04$ & $-0.10 \pm 0.02$ & $-0.030 \pm 0.006$ \\
27 Feb. 2003 & $B$ & $25.68 \pm 0.09$ & $-0.24 \pm 0.05$ & $0.046 \pm 0.022$ \\
& $R$ & $25.66 \pm 0.12$ & $-0.16 \pm 0.07$ & $-0.028 \pm 0.021$ \\
\hline
\end{tabular}

4 Feb. 2002 (UGC 1703, UGC 5944) were calibrated with shallow images obtained on 26 Feb. 2003.

$B$ and $R$ images of each galaxy were registered by matching the positions of typically 50 reference stars spread evenly over the image. The alignment was done on a pixel scale in order to avoid dividing galaxy flux in subpixel shifts. The resulting slight degradation of image quality is insignificant in relation to the seeing effects in the images. The sky background level was estimated by fitting a plane to selected star-free areas distributed uniformly over the CCD area but well away from the galaxy. The sky-subtracted images taken in the same passband were cleaned of cosmic rays and median-combined to increase the signal-to-noise. Finally, the resulting master images were flux calibrated.

\section{3. $R$-band SBF analysis of selected early-type dwarf galaxies in the $\mathbf{1 0} \mathrm{Mpc}$ range}

We applied the Surface Brightness Fluctuation (SBF) method, developed to measure distances to dwarf elliptical galaxies by Jerjen et al. (1998, 2000b) and calibrated in Jerjen et al. (2001). The SBF analysis is done by carefully cleaning the galaxy images from any foreground stars, globular clusters, and background galaxies using procedures that follow the recipes of Jerjen et al. (2000b, 2001). The cleaned galaxy image was then modelled using an isophote fitting routine written in IRAF that allows the centre, ellipticity, and position angle to vary. The best 2D-model was subtracted from the original master image and the residual image divided by the square root of the model for noise normalization.

Any non-radial irregularities in the light distribution of a galaxy such as the detected spiral arms and dust features in NGC 4150 remain visible in the residual image (see Fig. 2). These parts of a galaxy were avoided in the SBF field selection. Only regions where the model follows closely the galaxy light distribution were used.

The largest possible number of slightly overlapping square subimages (hereafter SBF fields) were then defined for each galaxy. The size of the SBF fields was chosen between $70 \times 70$ and $100 \times 100$ pixels depending on the apparent diameter of the galaxy. Parts of a SBF field that were contaminated by foreground stars or background galaxies were replaced with randomly selected patches from the fluctuation image, lying outside the field and in the same surface brightness range of the galaxy. The number of patched pixels was $3 \%$ or less of the total SBF field area in all cases. In total we defined 61 SBF fields in our 10 sample galaxies. Their positions across the galaxy images are shown in Fig. 1.

All SBF fields were Fourier transformed and the azimuthally averaged power spectra calculated. From isolated bright stars on the galaxy master image we determined the point spread function (PSF) profile. We then fitted a linear combination of the flux normalized and exposure time weighted PSF power spectrum and a constant at the observed galaxy power spectrum $\operatorname{PS}(k)=P_{0} \mathrm{PS}_{\text {star }}(k)+P_{1}$, demanding a least squares minimization. Data points at low spatial frequencies $(k \leq 5)$ were omitted as they are likely affected by imperfect galaxy model subtraction. Figures 3 to 9 and 11 to 13 show the power spectrum of each SBF field with the best fitting analytic function indicated as solid lines. Table 4 summarizes the quantities measured in the SBF analysis: Col. 1 - SBF field number and galaxy name, Col. 2 - pixel size of the SBF field, Col. 3 - magnitude $m_{1}$ of a star yielding 1 ADU per second on the CCD, Col. 4 - mean galaxy surface brightness within the SBF field in ADU, Col. 5 - sky brightness in ADU, Col. 6 - exposure time normalized amplitude $P_{0}$ of the best least squares fit at wavenumber $k=0$ with fitting error in brackets, Col. 7 - the scale-free white noise component $P_{1}$ in the power spectrum, indicating the ratio of sky to mean galaxy surface brightness within the SBF field.

To estimate the fraction in $P_{0}$ (Col. 6) from unresolved distant background galaxies fainter than the cutoff magnitude $m_{\mathrm{c}}=24.7 R \mathrm{mag}$, we made use of a formula that was given in Jensen et al. (1998) and adjusted for the $R$-band by Jerjen et al. (2001):

$P_{\mathrm{BG}}=\frac{p^{2}}{(0.8-\gamma) \ln 10} 10^{0.8\left(m_{1}-m_{\mathrm{c}}\right)-\gamma\left(29.38+R-K-m_{\mathrm{c}}\right)}$,

where $p$ is the CCD pixel size in arcsec and $\gamma=0.3$ the slope of the power-law number distribution for background galaxies in the $K$-band (Cowie et al. 1994). Assuming a typical galaxy colour of $(R-K)=2.25$ (de Jong 1996) we computed $P_{\mathrm{BG}}$ and determined the signal-to-noise $S / N=\left(P_{0}-P_{\mathrm{BG}}\right) /\left(P_{1}+P_{\mathrm{BG}}\right)$ as well as the relative contribution to the signal $P_{\mathrm{BG}} / P_{0}$ for each individual SBF field. Both numbers are listed in Col. 8 and Col. 9 of Table 4. The contribution from unresolved background galaxies was minimal at the 0-6 per cent level in our SBF fields.

Another potentially significant source of unwanted fluctuations is a rich globular cluster (GC) system in a target galaxy. While this is an important issue for luminous giant ellipticals the expected number of GCs in our dwarf ellipticals is quite small. For instance, the net number of globular cluster candidates for UGC 5944 in the Leo Group is $3.5 \pm 3.9$ (Miller et al. 1998). The GC frequency $\left(S_{N}\right)$-luminosity relation for dE,Ns studied in the Fornax and Virgo clusters (Miller et al. 1998) predicts $\approx 18$ GCs (assuming $S_{N}=2$ ) for our brightest dwarf NGC $4150\left(M_{V} \approx-17.4\right)$ and $\approx 0$ GCs (assum$\left.\operatorname{ing} S_{N}=10\right)$ for the faintest dwarf UGC $5442\left(M_{V} \approx-10\right)$. All GCs would be brighter than our cutoff luminosity and thus be excised during the image cleaning process. Therefore, no further corrections were applied to the measured SBF power. 


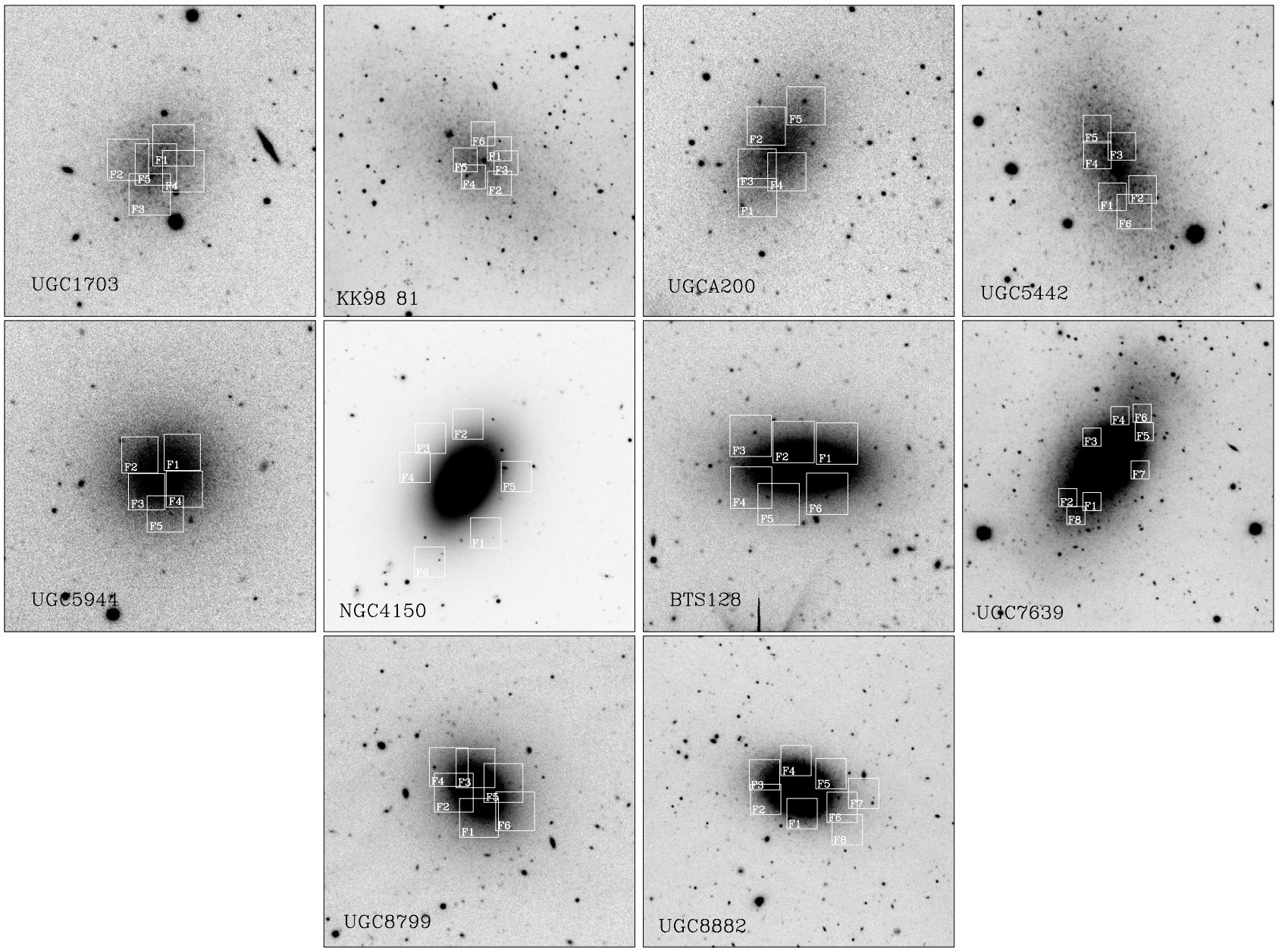

Fig. 1. Reduced $R$-band images of the ten sample galaxies with the boundaries of the analysed square SBF fields indicated. The field-of-view is $2.5 \times 2.5 \mathrm{arcmin}$. North is up, East to the left.

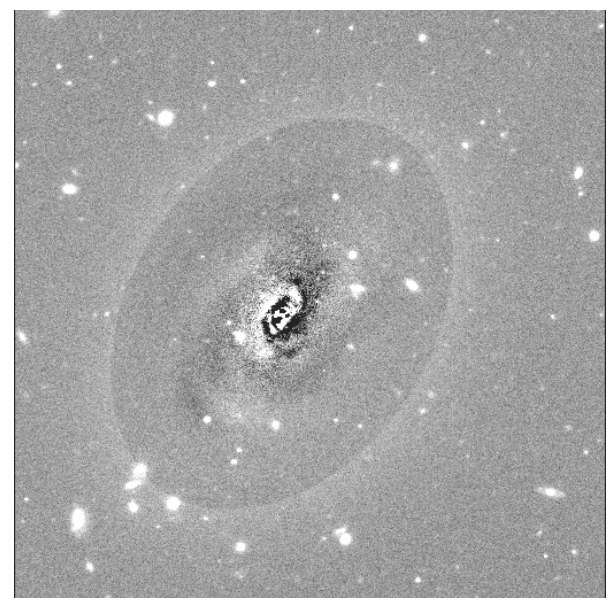

Fig. 2. After subtracting the best-fitting model of the global light distribution a well developed 4-armed spiral structure and traces of dust in the central region become visible in the residual image of NGC 4150. These findings confirm the morphological type of S0/Sa for this galaxy.

Finally, we calculated the stellar fluctuation magnitude $\bar{m}_{R}$ with the formula $\bar{m}_{R}=m_{1}-2.5 \log \left(P_{0}-P_{\mathrm{BG}}\right)$ and measured the $(B-R)$ colour for each SBF field from the cleaned $B$ and $R$ galaxy master images. Both quantities were corrected for foreground extinction using the IRAS/DIRBE maps of dust IR emission (Schlegel et al. 1998). The results are listed in Cols. 3 and 4 of Table 5.

The power spectrum fitting error is between 3 and $15 \%$. Other sources of minor errors are the PSF normalization $(\sim 2 \%)$, the shape variation of the stellar PSF over the CCD area (1-2\%) and the uncertainty in the photometric calibration ( $0.04 \mathrm{mag}$ in $B, 0.03 \mathrm{mag}$ in $R$ ). If we further adopt a $16 \%$ error for the foreground extinction (Schlegel et al. 1998), the formal combined error for a single $\bar{m}_{R}^{0}$ measurement is between 0.05 and $0.20 \mathrm{mag}$ (Col. 3). The error associated with the local colour (Col. 4) has been obtained through the usual error propagation formula from the uncertainties in the sky level determination, the photometry zero points, and Galactic extinction.

\section{Discussion}

\section{UGC 1703}

Neither a velocity nor a distance were known for UGC 1703 to date. This dwarf galaxy is closest in projection (3 degrees) to the spiral galaxy NGC 925 for which a Cepheid distance 

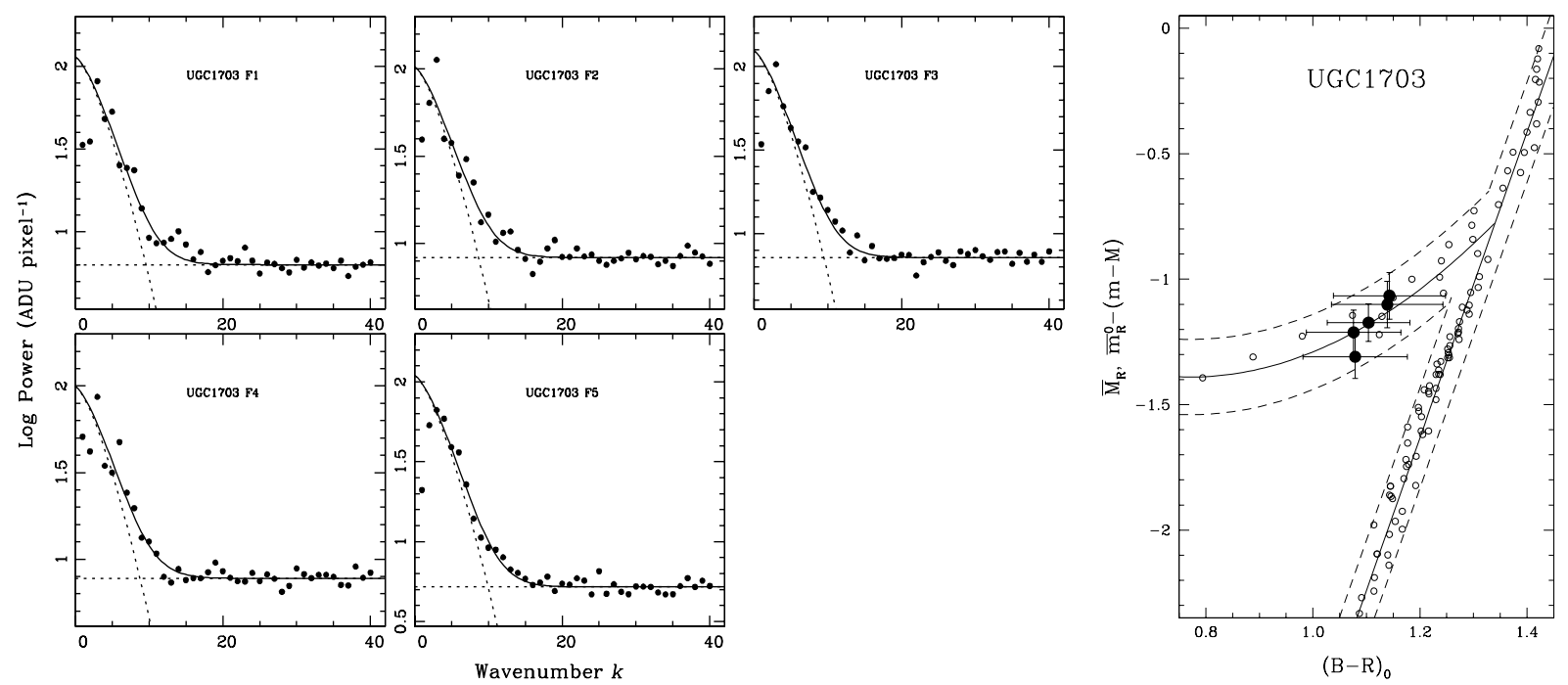

Fig. 3. Left: five fields were selected for the SBF analysis in UGC 1703. The signal-to-noise in the power spectra is generally high. The power spectra of the SBF fields (filled circles) are well fitted by the sum (solid line) of a scaled version of the power spectrum of the PSF and a constant (dashed lines). The wavenumbers 1-4 were not considered for the fit. Right: the fluctuation magnitudes $\bar{m}_{R}$ and $(B-R)$ colours were measured for the five fields to derive the distance of UGC 1703. A shift by 28.11 mag yields the best fit of the data to the calibration diagram.
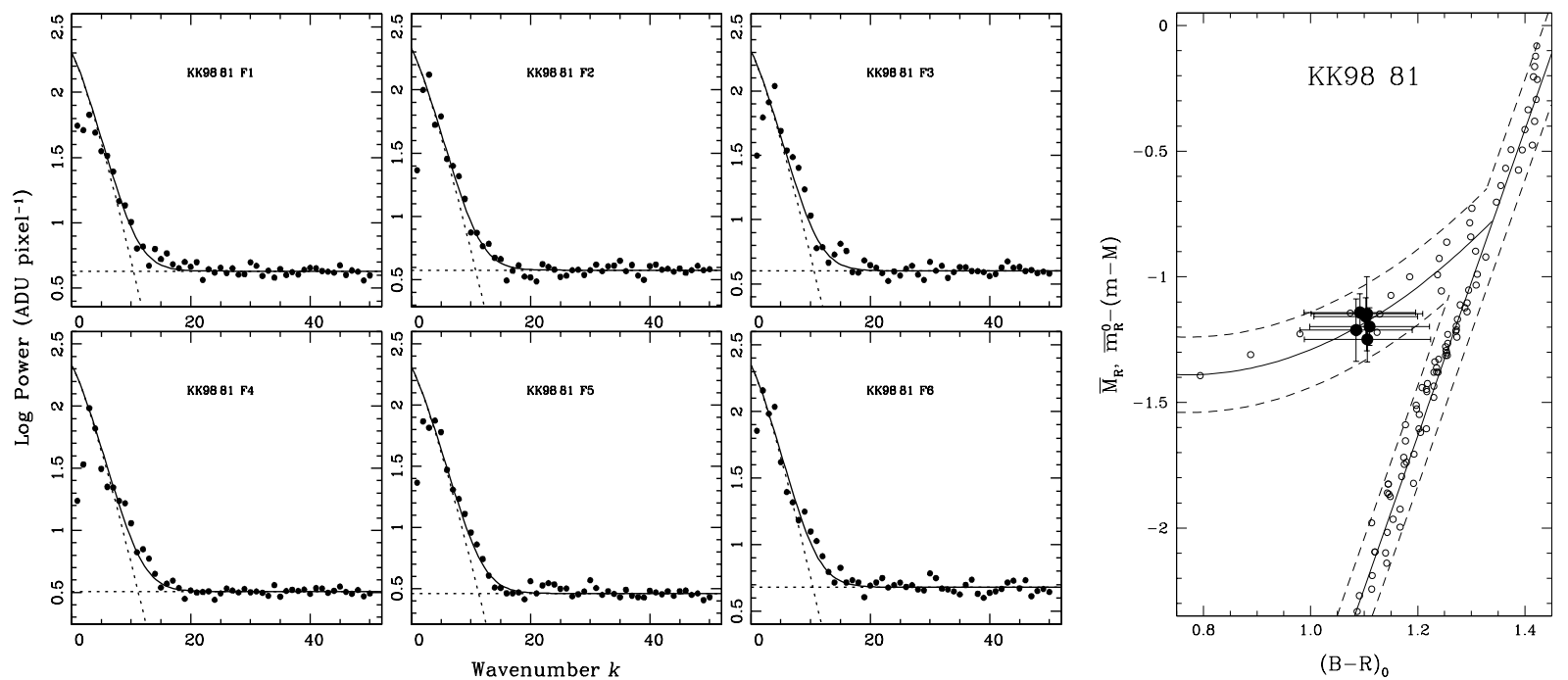

Fig. 4. Left: six fields were selected for the SBF analysis in KDG 61. The power spectra of the SBF fields (filled circles) are well fitted by the sum (solid line) of a scaled version of the power spectrum of the PSF and a constant (dashed lines). The wavenumbers $1-4$ were not considered for the fit. Right: the fluctuation magnitudes $\bar{m}_{R}$ and $(B-R)$ colours were measured for the six fields to derive the distance of KDG 61. A shift by $27.80 \mathrm{mag}$ yields the best fit of the data to the calibration diagram.

of $9.3 \pm 0.7 \mathrm{Mpc}$ (Silbermann et al. 1996) and a velocity of $v_{\odot}=553 \mathrm{~km} \mathrm{~s}^{-1}$ were reported. However, our SBF distance for UGC 1703 of $4.2 \pm 0.3 \mathrm{Mpc}$ indicates a much shorter distance from the Milky Way and thus UGC 1703 seems to be only close to NGC 925 in projection. The next nearest major galaxy to UGC 1703 is the SBdm spiral NGC 784 with an angular separation of 4.7 degrees. NGC 784 has a velocity of $v_{\odot}=$ $198 \mathrm{~km} \mathrm{~s}^{-1}$ and a reported distance of $5 \mathrm{Mpc}$ (Drozdovsky \& Karachentsev 2000). These two results confirm that UGC 1703 is spatially close to NGC 784 with a projected linear distance of only $\sim 0.4 \mathrm{Mpc}$ at an adopted distance of $4.5 \mathrm{Mpc}$. Another more qualitative evidence for the short distance of UGC 1703 comes from our deep $R$-band image (Fig. 1) which shows semi-resolved stars in appearance similar to images of dwarf ellipticals in the Cen A Group observed with a $2.3 \mathrm{~m}$ groundbased telescope (Jerjen et al. 2000a).

\section{$K D G 61([K K 98] 81)$}

KDG 61 ([KK98] 81) is a member of the M 81 group. Karachentsev et al. (2000) reported a distance modulus of $(m-M)=27.78 \pm 0.15 \mathrm{mag}$ from the measurement of the magnitude of the red giant branch tip and the galaxy has a heliocentric velocity of $v_{\odot}=-135 \pm 30 \mathrm{~km} \mathrm{~s}^{-1}$ (Johnson et al. 1997). We analysed six independent SBF fields across the galaxy's surface (Fig. 4, left panels). The derived mean distance modulus of $(m-M)_{\mathrm{SBF}}=27.80 \pm 0.20 \mathrm{mag}$ (see Fig. 4, right panel) is in good agreement with the TRGB result. Due to the small 

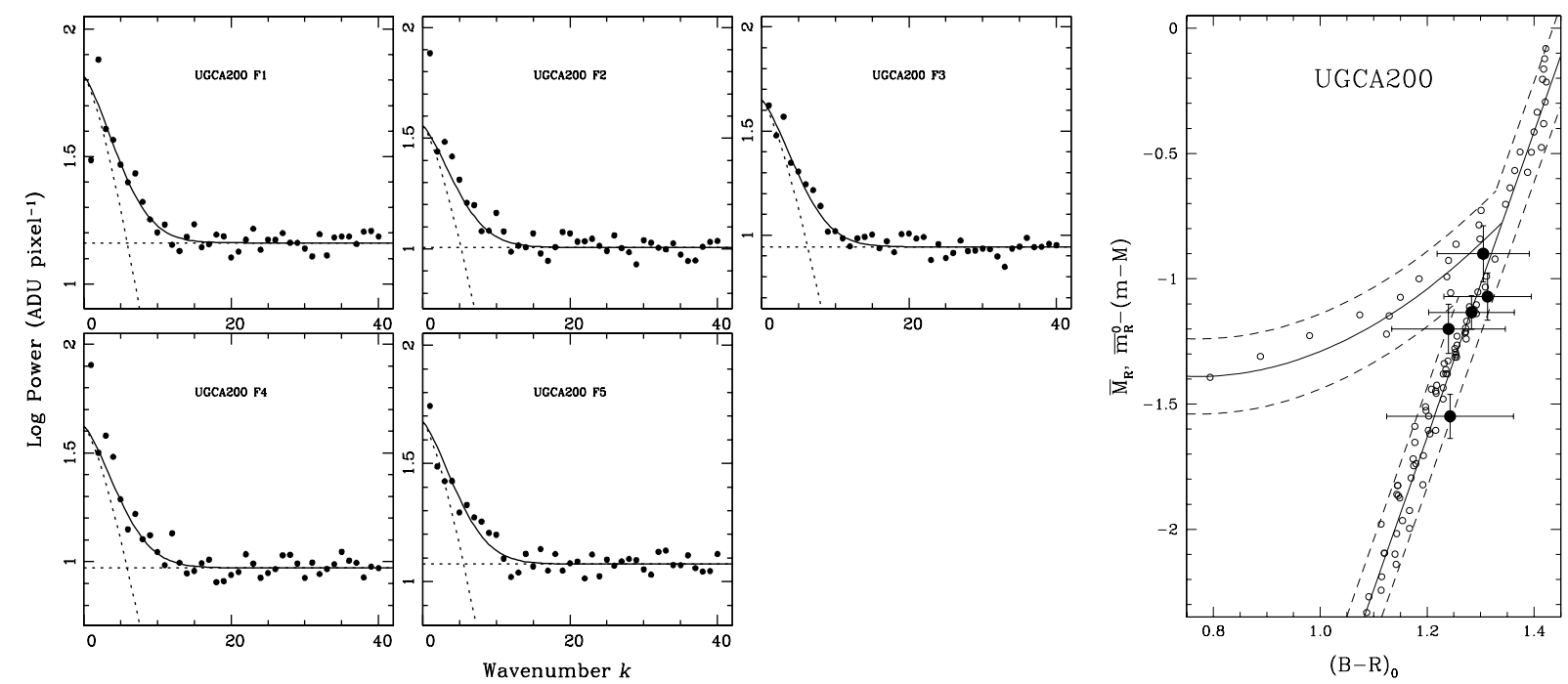

Fig. 5. Left: five fields were selected for the SBF analysis in UGCA 200. The power spectra of the SBF fields (filled circles) are well fitted by the sum (solid line) of a scaled version of the power spectrum of the PSF and a constant (dashed lines). The wavenumbers 1-4 were not considered for the fit. Right: the fluctuation magnitudes $\bar{m}_{R}$ and $(B-R)$ colours were measured for the five fields to derive the distance of UGCA 200. A shift by 29.01 mag yields the best fit of the data to the linear branch of the calibration diagram.
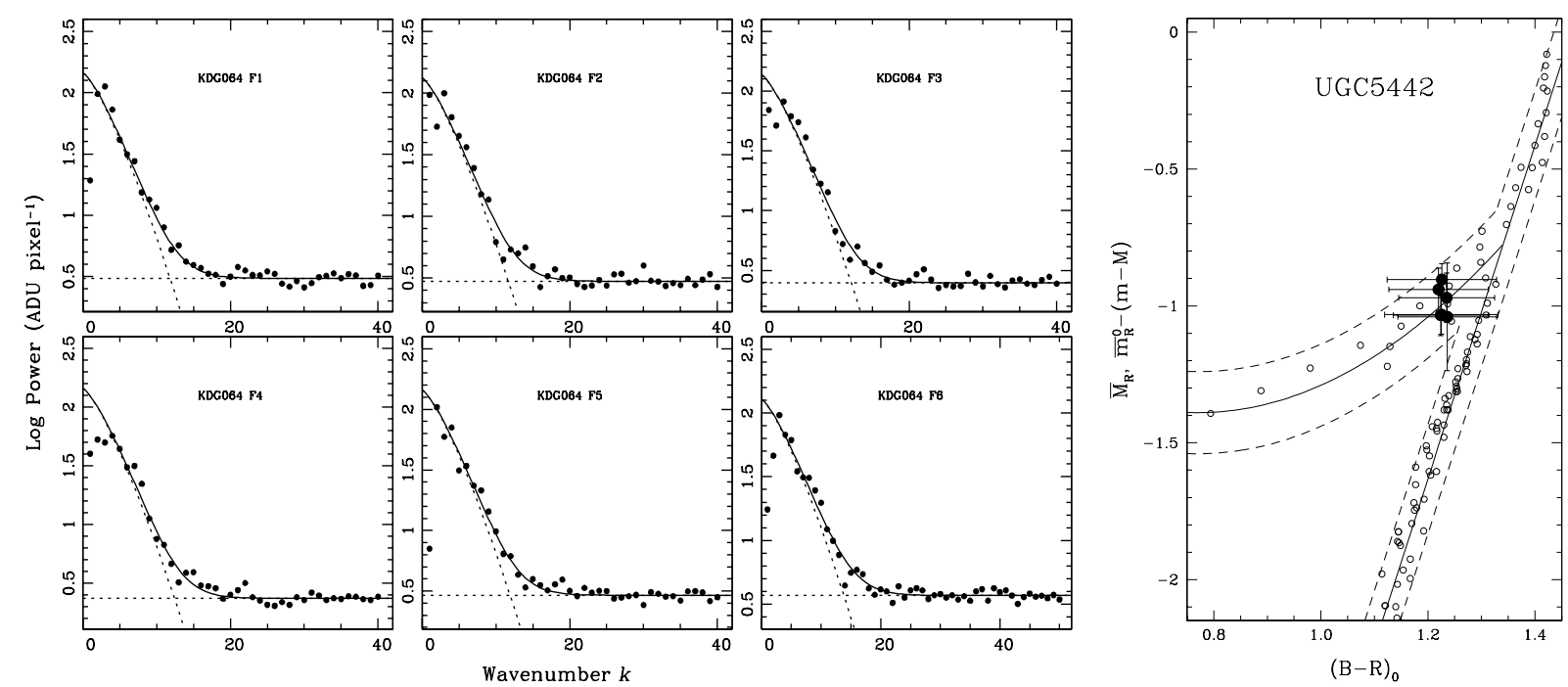

Fig. 6. Left: six fields were selected for the SBF analysis in UGC 5442. The power spectra of the SBF fields (filled circles) are well fitted by the sum (solid line) of a scaled version of the power spectrum of the PSF and a constant (dashed lines). The wavenumbers 1-4 were not considered for the fit. Right: six fields were analysed to derive the distance of UGC 5442. A shift by 27.74 mag yields the best fit of the data to the calibration diagram.

colour range covered by the SBF fields (see Table 5), another SBF distance is technically possible by moving the data points onto the linear branch of the calibration curve. However, that alternative distance modulus of $(m-M)_{\mathrm{SBF}}=30.0 \pm 0.3 \mathrm{mag}$ is highly inconsistent with the independent TRGB result and thus can be ruled out.

\section{UGCA 200}

Neither a velocity nor a distance were known for UGCA 200 to date. This dE,N was previously photometrically studied by Parodi et al. (2002). The authors reported an outward colour gradient getting redder and an integrated colour of
$(B-R)_{0}=1.38$. Our five SBF fields were selected from the inner region of the dwarf and thus are slightly bluer, in the range $1.22<(B-R)_{0}<1.31$. Nevertheless, the SBF fields of UGCA 200 are the reddest in our sample. The observed correlation between the derived parameters $\bar{m}_{R}$ and $(B-R)$ colour allowed an unambiguous measurement of the distance. A shift by 29.01 mag yields the best fit to the linear branch of the calibration diagram. UGCA 200 was assumed to be a faint companion of the S0 galaxy NGC 3115 which has a velocity of $v_{\odot}=720 \mathrm{~km} \mathrm{~s}^{-1}$ and an accurate SBF distance of $9.7 \pm 0.1 \mathrm{Mpc}$ (Tonry et al. 2001). However, our SBF distance for UGCA 200 is significantly shorter with $6.3 \pm 0.8 \mathrm{Mpc}$ and thus suggests that this dwarf is actually in the foreground of NGC 3115. 

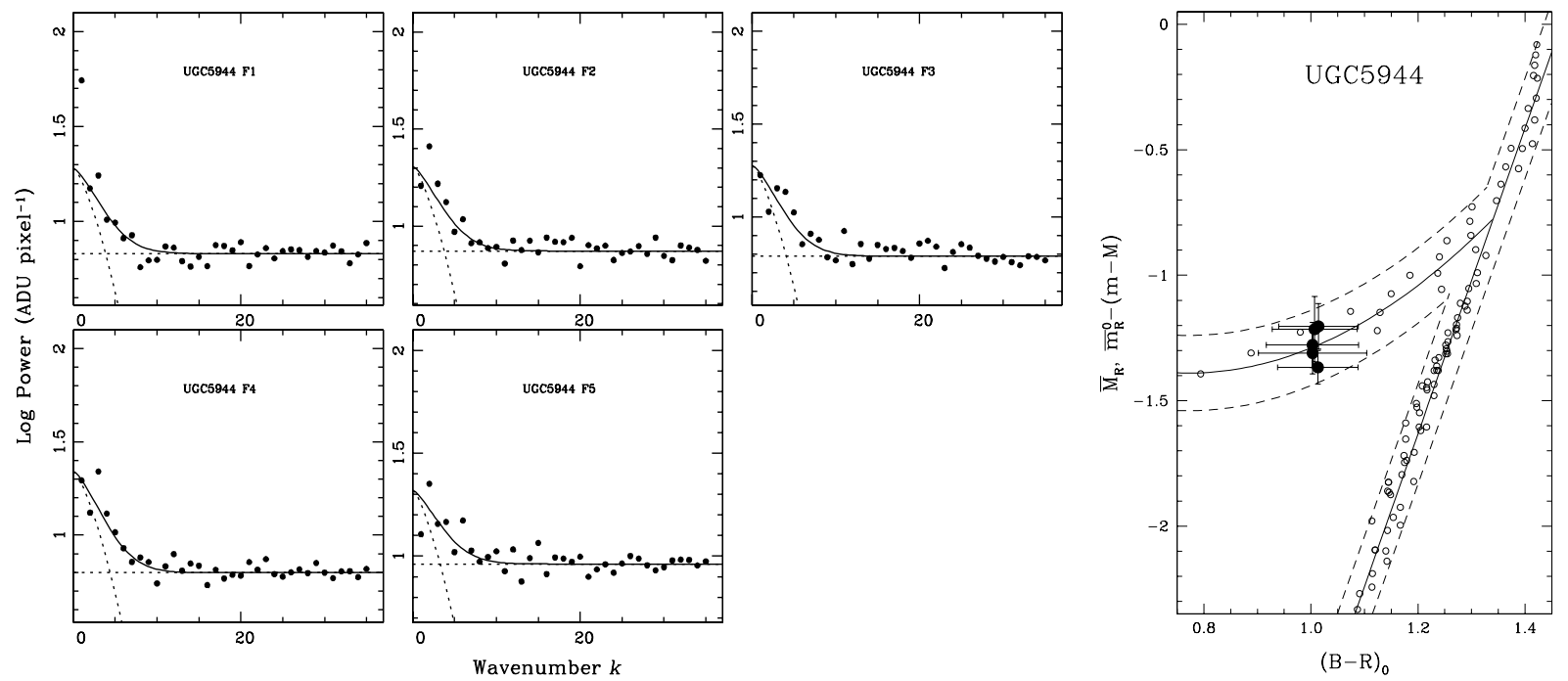

Fig. 7. Left: five fields were selected for the SBF analysis in UGC 5944. The signal-to-noise in the power spectra is not as high as measured in the other galaxies due to the larger distance of the galaxy at $11.1 \mathrm{Mpc}$. Nevertheless, the power spectra of the SBF fields (filled circles) are well fitted by the sum (solid line) of a scaled version of the power spectrum of the PSF and a constant (dashed lines). The wavenumbers $1-4$ were not considered for the fit. Right: five fields were analysed to derive the distance of UGC 5944. A shift by 30.22 mag yields the best fit of the data to the calibration diagram.
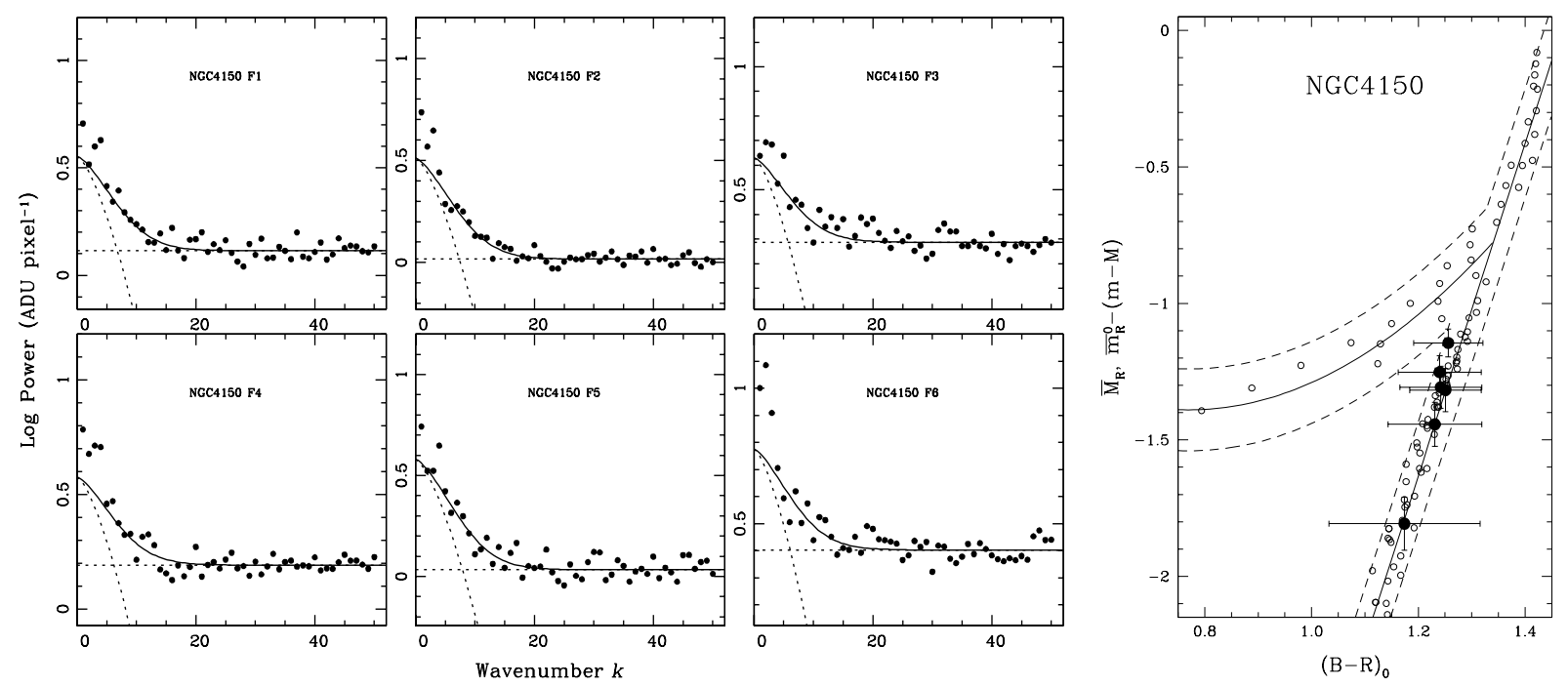

Fig. 8. Left: six fields were selected for the SBF analysis in NGC 4150. The signal-to-noise in the power spectra is not as high as measured in the other galaxies, as it is more distant. Nevertheless, the power spectra of the SBF fields (filled circles) are well fitted by the sum (solid line) of a scaled version of the power spectrum of the PSF and a constant (dashed lines). The wavenumbers 1-4 were not considered for the fit. Right: six fields were analysed to derive the distance of NGC 4150. A shift by 30.79 mag yields the best fit of the data to the calibration diagram.

\section{UGC 5442 (KDG 64)}

UGC 5442 (KDG 64) is a member of the M 81 group. Karachentsev et al. (2000) reported a distance modulus of $(m-M)=27.84 \pm 0.15 \mathrm{mag}$ from the measurement of the magnitude of the red giant branch tip. The galaxy has a heliocentric velocity of $v_{\odot}=-18 \pm 14 \mathrm{~km} \mathrm{~s}^{-1}$ (Simien \& Prugniel 2002). We analysed six independent SBF fields across the galaxy's surface (Fig. 6, left panels). The derived mean distance modulus of $(m-M)_{\mathrm{SBF}}=27.74 \pm 0.20 \mathrm{mag}$ (see Fig. 6, right panel) is in good agreement with the TRGB result. Due to the small colour range covered by the SBF fields (see Table 5), another SBF distance is technically possible by moving the data points onto the linear branch of the calibration curve. However, the alternative distance modulus of $(m-M)_{\mathrm{SBF}}=$ $28.21 \pm 0.3 \mathrm{mag}$ is inconsistent with the TRGB result.

\section{UGC 5944 ([FS90] 047)}

Neither a velocity nor a distance were known for UGC 5944 to date. This dwarf elliptical galaxy was catalogued as member of the Leo Group in Ferguson \& Sandage (1990) based on its morphology. With our SBF distance of $11.1 \pm 0.9 \mathrm{Mpc}$ we can now confirm this impression. The derived SBF distance is also in good agreement with the mean distance of $11 \mathrm{Mpc}$ for the group (Trentham \& Tully 2002). We note that there was not 

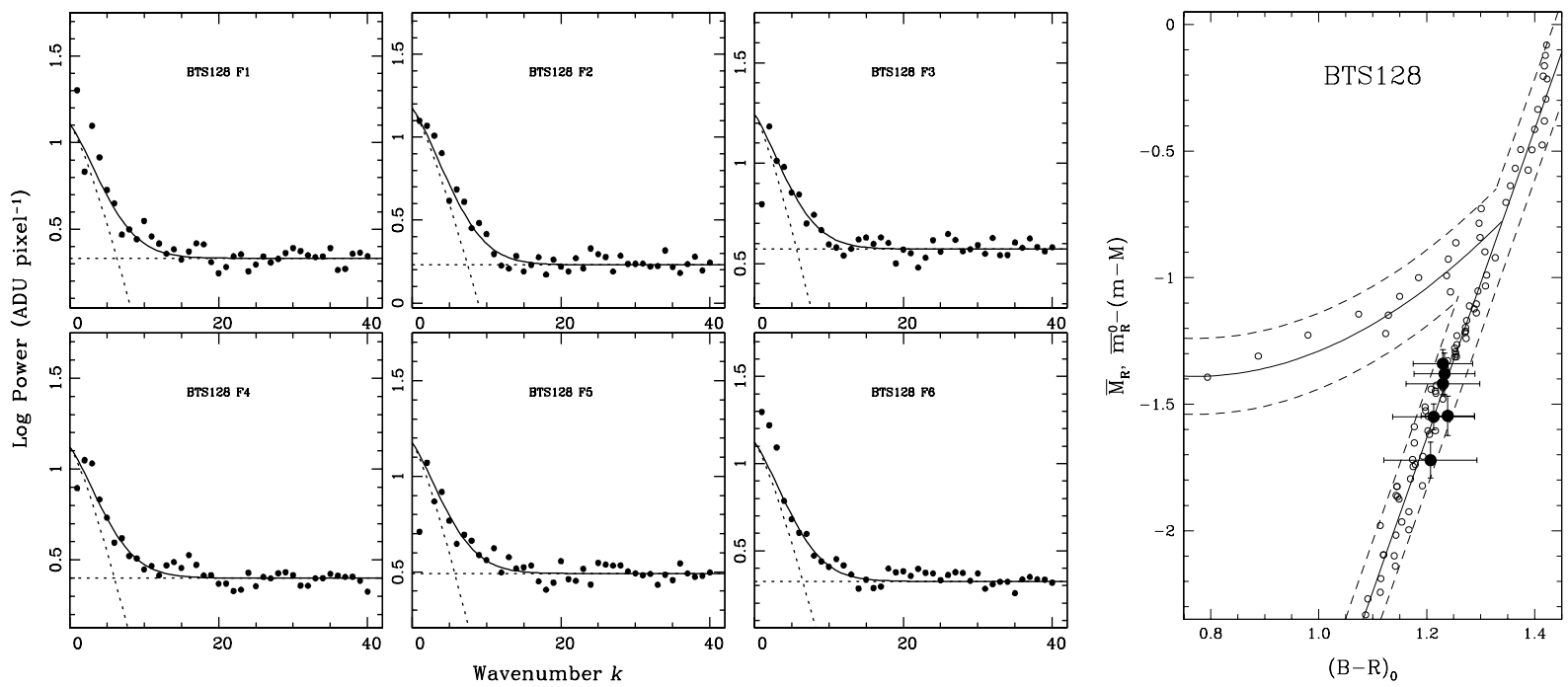

Fig. 9. Left: six fields were selected for the SBF analysis in BTS 128. The power spectra of the SBF fields (filled circles) are well fitted by the sum (solid line) of a scaled version of the power spectrum of the PSF and a constant (dashed lines). The wavenumbers 1-4 were not considered for the fit. Right: six fields were analysed to derive the distance of BTS 128. A shift by 31.02 mag yields the best fit of the data to the calibration diagram.

much of colour range found in the five fields we analysed to unambiguously apply the distance calibration. Consequently, there is an alternative distance of $\approx 22 \mathrm{Mpc}$ for this galaxy. However, this latter distance appears less likely.

\section{NGC 4150}

NGC 4150 is morphologically classified as S0/Sa galaxy (Sandage \& Bedke 1994). Once the galaxy light model is subtracted, the residual image shows a prominent, well developed 4-armed spiral structure with non-circular dust pattern in the central region (Fig. 2). The galaxy has a heliocentric velocity of $v_{\odot}=226 \pm 22 \mathrm{~km} \mathrm{~s}^{-1}$ (Fisher et al. 1995). Tonry et al. (2001) reported a first SBF distance modulus of $(m-M)=30.69 \pm 0.25 \mathrm{mag}$ (or $13.7 \pm 1.7 \mathrm{Mpc}$ ) and Jensen et al. (2003) another, corrected SBF distance modulus of $(m-M)=30.53 \pm 0.24 \mathrm{mag}$ (or $12.8 \pm 1.5 \mathrm{Mpc}$ ). We analysed six independent SBF fields across the galaxy's surface but well away from the spiral structures (Fig. 2). The derived distance modulus of $(m-M)_{\mathrm{SBF}}=30.79 \pm 0.20 \mathrm{mag}$ (see Fig. 8) agrees with the earlier published SBF results.

\section{BTS 128}

This dwarf elliptical is located right in the densest region of the Coma I group (Binggeli et al. 1990; Trentham \& Tully 2002) about 10 degrees away from the northern boundary of the Virgo cluster. The heliocentric velocity of BTS 128 of $v_{\odot}=1139 \pm 86 \mathrm{~km} \mathrm{~s}^{-1}$ (Wegner et al. 2001) is in good agreement with the Coma I cluster. BTS 128 is 27.5 arcmin away from the E0 galaxy NGC 4283 for which a SBF distance of $(m-M)=30.98 \pm 0.19$ mag was reported by Tonry et al. (2001). The SBF distance module we derive for BTS 128 is $(m-M)_{\mathrm{SBF}}=31.02 \pm 0.25 \mathrm{mag}(16.0 \pm 2.0 \mathrm{Mpc})$.

\section{UGC 7639}

This galaxy, classified as $\mathrm{dE} / \mathrm{Im}$, is located in the Canes Venatici region. The Im nature of the otherwise featureless dwarf galaxy becomes visible once the galaxy model is subtracted, with a central region of bright young stars and evidence of dust (see Fig. 10). It has a heliocentric velocity of $v_{\odot}=$ $382 \mathrm{~km} \mathrm{~s}^{-1}$ (de Vaucouleurs et al. 1991). Makarova et al. (1998) published a rough estimate of the distance of $8.0 \mathrm{Mpc}$ based on brightest blue $(B-V<0.4)$ and red $(B-V>1.6)$ stars. We analysed eight independent SBF fields across the galaxy's surface (Figs. 10 and 11, left panels). Because of the absence of a steep correlation between colour and SBF magnitude for the different SBF fields, we can use unambiguously the parabolic branch of the calibration diagram. The resulting SBF distance of $7.1 \pm 0.6 \mathrm{Mpc}$ or $(m-M)_{\mathrm{SBF}}=29.27 \pm 0.16 \mathrm{mag}$ (see Fig. 11, right panel) identifies this galaxy as slightly closer than previously thought.

\section{UGC 8799}

No distance was known for this dwarf elliptical to date but Geller et al. (1997) and Wegner et al. (2001) report a velocity of $1132 \mathrm{~km} \mathrm{~s}^{-1}$. Because this galaxy is only $1.5 \mathrm{~h}$ in RA from the western boundary of the Virgo cluster and the velocity agrees well with the mean cluster velocity $\left(980 \pm 60 \mathrm{~km} \mathrm{~s}^{-1}\right.$, Tanaka 1985) this may suggest that UGC 8799 has a distance that falls into the observed distance range of the Virgo cluster $14 \mathrm{Mpc}<D<23 \mathrm{Mpc}$ (Jerjen et al. 2004). The six SBF fields (see Fig. 12) show only a narrow $B-R$ colour spread and the signal-to-noise in the power spectra is low. This leads to two possible distance moduli: $(m-M)_{\mathrm{SBF}}=30.19 \pm 0.23 \mathrm{mag}$ or $(m-M)_{\mathrm{SBF}}=30.61 \pm 0.26 \mathrm{mag}$ (see Fig. 12), suggesting it is in the outskirts of the Virgo cluster. 
Table 4. Parameters of the SBF analysis.

\begin{tabular}{|c|c|c|c|c|c|c|c|c|}
\hline $\begin{array}{c}\text { Name } \\
\text { (1) }\end{array}$ & $\begin{array}{c}\text { Size } \\
\text { (pixels) } \\
(2)\end{array}$ & $\begin{array}{c}m_{1} \\
(\mathrm{mag}) \\
(3)\end{array}$ & $\begin{array}{c}\bar{g} \\
(\mathrm{ADU}) \\
(4)\end{array}$ & $\begin{array}{c}s \\
(\mathrm{ADU}) \\
(5)\end{array}$ & $\begin{array}{c}P_{0} \\
\left(\mathrm{ADU} \mathrm{s}^{-1} \mathrm{pixel}^{-1}\right) \\
(6)\end{array}$ & $\begin{array}{c}P_{1} \\
\left(\mathrm{ADU} \mathrm{s}^{-1} \mathrm{pixel}^{-1}\right) \\
(7)\end{array}$ & $\begin{array}{l}S / N \\
(8)\end{array}$ & $\begin{array}{c}P_{\mathrm{BG}} / P_{0} \\
\text { (9) }\end{array}$ \\
\hline UGC $1703 \mathrm{~F} 1$ & 80 & 25.34 & 80.4 & 2314.5 & $0.189(0.009)$ & 0.011 & 15.9 & 0.00 \\
\hline$\ldots \ldots \ldots$ F2 & 80 & & 66.8 & & $0.171(0.007)$ & 0.014 & 11.5 & 0.00 \\
\hline$\ldots \ldots \ldots$ F3 & 80 & & 70.6 & & $0.207(0.007)$ & 0.012 & 16.1 & 0.00 \\
\hline$\ldots \ldots \ldots$ F4 & 80 & & 66.1 & & $0.165(0.007)$ & 0.013 & 11.9 & 0.01 \\
\hline$\ldots \ldots \ldots$ F5 & 80 & & 99.1 & & $0.183(0.007)$ & 0.009 & 18.5 & 0.00 \\
\hline KDG $61 \mathrm{~F} 1$ & 100 & 25.47 & 68.2 & 1281.5 & $0.334(0.018)$ & 0.007 & 41.3 & 0.00 \\
\hline$\ldots \ldots \ldots \ldots \mathrm{F} 2$ & 100 & & 63.6 & & $0.352(0.018)$ & 0.006 & 49.7 & 0.00 \\
\hline$\ldots \ldots \ldots$ F3 & 100 & & 68.7 & & $0.336(0.047)$ & 0.007 & 41.6 & 0.00 \\
\hline$\ldots \ldots \ldots \ldots \mathrm{F} 4$ & 100 & & 67.8 & & $0.357(0.040)$ & 0.005 & 58.8 & 0.00 \\
\hline$\ldots \ldots \ldots$ F5 & 100 & & 76.0 & & $0.339(0.019)$ & 0.005 & 55.8 & 0.00 \\
\hline$\ldots \ldots \ldots$ F6 & 100 & & 59.5 & & $0.369(0.026)$ & 0.008 & 40.6 & 0.00 \\
\hline UGCA $200 \mathrm{~F} 1$ & 80 & 25.17 & 63.6 & 2534.0 & $0.109(0.003)$ & 0.024 & 4.4 & 0.01 \\
\hline$\ldots \ldots \ldots \mathrm{F} 2$ & 80 & & 98.8 & & $0.060(0.006)$ & 0.017 & 3.4 & 0.01 \\
\hline$\ldots \ldots \ldots$ F3 & 80 & & 107.4 & & $0.074(0.003)$ & 0.015 & 4.7 & 0.01 \\
\hline$\ldots \ldots \ldots$ F4 & 80 & & 107.4 & & $0.070(0.005)$ & 0.016 & 4.2 & 0.01 \\
\hline$\ldots \ldots \ldots$ F5 & 80 & & 72.7 & & $0.079(0.005)$ & 0.020 & 3.8 & 0.01 \\
\hline UGC 5442 F1 & 80 & 25.30 & 107.6 & 1441.1 & $0.243(0.047)$ & 0.005 & 42.0 & 0.00 \\
\hline$\ldots \ldots \ldots \mathrm{F} 2$ & 80 & & 105.2 & & $0.221(0.015)$ & 0.005 & 38.1 & 0.00 \\
\hline$\ldots \ldots \ldots \mathrm{F} 3$ & 80 & & 111.7 & & $0.227(0.018)$ & 0.004 & 47.4 & 0.00 \\
\hline$\ldots \ldots \ldots$ F4 & 80 & & 113.6 & & $0.241(0.015)$ & 0.004 & 50.3 & 0.00 \\
\hline$\ldots \ldots \ldots$ F5 & 80 & & 87.2 & & $0.241(0.013)$ & 0.005 & 41.6 & 0.00 \\
\hline$\ldots \ldots \ldots$ F6 & 100 & & 91.4 & & $0.214(0.009)$ & 0.006 & 31.5 & 0.00 \\
\hline UGC 5944 F1 & 70 & 25.34 & 172.7 & 2143.7 & $0.032(0.004)$ & 0.011 & 2.6 & 0.03 \\
\hline$\ldots \ldots \ldots F \mathrm{~F} 2$ & 70 & & 148.6 & & $0.034(0.003)$ & 0.012 & 2.6 & 0.02 \\
\hline$\ldots \ldots \ldots$ F3 & 70 & & 195.7 & & $0.032(0.003)$ & 0.010 & 2.9 & 0.03 \\
\hline$\ldots \ldots \ldots$ F4 & 70 & & 192.0 & & $0.037(0.002)$ & 0.010 & 3.3 & 0.02 \\
\hline$\ldots \ldots \ldots$ F5 & 70 & & 117.9 & & $0.035(0.002)$ & 0.015 & 2.2 & 0.02 \\
\hline NGC $4150 \mathrm{~F} 1$ & 100 & 25.33 & 94.5 & 388.7 & $0.020(0.001)$ & 0.007 & 2.5 & 0.04 \\
\hline$\ldots \ldots \ldots \mathrm{F} 2$ & 100 & & 141.3 & & $0.018(0.001)$ & 0.006 & 2.5 & 0.05 \\
\hline$\ldots \ldots \ldots$ F3 & 100 & & 77.0 & & $0.024(0.002)$ & 0.011 & 2.0 & 0.03 \\
\hline$\ldots \ldots \ldots$ F4 & 100 & & 97.7 & & $0.021(0.002)$ & 0.009 & 2.1 & 0.04 \\
\hline$\ldots \ldots \ldots$ F5 & 100 & & 129.2 & & $0.021(0.002)$ & 0.006 & 3.0 & 0.04 \\
\hline$\ldots \ldots \ldots$ F6 & 100 & & 40.7 & & $0.033(0.003)$ & 0.014 & 2.2 & 0.02 \\
\hline BTS $128 \mathrm{~F} 1$ & 80 & 25.52 & 174.1 & 1050.0 & $0.021(0.002)$ & 0.004 & 3.8 & 0.06 \\
\hline$\ldots \ldots \ldots \mathrm{F} 2$ & 80 & & 243.8 & & $0.025(0.002)$ & 0.003 & 5.7 & 0.05 \\
\hline$\ldots \ldots \ldots \mathrm{F} 3$ & 80 & & 85.6 & & $0.029(0.002)$ & 0.006 & 3.9 & 0.04 \\
\hline$\ldots \ldots \ldots \mathrm{F} 4$ & 80 & & 120.8 & & $0.022(0.001)$ & 0.004 & 4.0 & 0.05 \\
\hline$\ldots \ldots \ldots$ F5 & 80 & & 102.7 & & $0.025(0.001)$ & 0.005 & 3.9 & 0.05 \\
\hline$\ldots \ldots \ldots$ F6 & 80 & & 183.5 & & $0.022(0.001)$ & 0.004 & 4.0 & 0.05 \\
\hline UGC 7639 F1 & 70 & 25.52 & 220.8 & 1157.9 & $0.105(0.005)$ & 0.002 & 32.9 & 0.01 \\
\hline$\ldots \ldots \ldots \mathrm{F} 2$ & 70 & & 148.3 & & $0.098(0.006)$ & 0.003 & 23.3 & 0.01 \\
\hline$\ldots \ldots \ldots \mathrm{F} 3$ & 70 & & 170.0 & & $0.110(0.006)$ & 0.003 & 26.2 & 0.01 \\
\hline$\ldots \ldots \ldots$ F4 & 70 & & 133.7 & & $0.098(0.005)$ & 0.003 & 23.3 & 0.01 \\
\hline$\ldots \ldots \ldots$ F5 & 70 & & 163.1 & & $0.112(0.006)$ & 0.003 & 26.7 & 0.01 \\
\hline$\ldots \ldots \ldots$ F6 & 70 & & 117.4 & & $0.111(0.008)$ & 0.004 & 21.3 & 0.01 \\
\hline$\ldots \ldots \ldots$ F7 & 70 & & 164.8 & & $0.096(0.005)$ & 0.004 & 18.4 & 0.01 \\
\hline$\ldots \ldots \ldots \ldots$ F8 & 70 & & 124.3 & & $0.102(0.006)$ & 0.003 & 24.2 & 0.01 \\
\hline UGC $8799 \mathrm{~F} 1$ & 100 & 25.32 & 184.7 & 1695.7 & $0.030(0.004)$ & 0.004 & 5.6 & 0.04 \\
\hline$\ldots \ldots \ldots \mathrm{F} 2$ & 100 & & 197.9 & & $0.029(0.003)$ & 0.004 & 5.9 & 0.03 \\
\hline$\ldots \ldots \ldots \mathrm{F} 3$ & 100 & & 209.9 & & $0.027(0.002)$ & 0.004 & 5.5 & 0.03 \\
\hline$\ldots \ldots \ldots F \mathrm{~F} 4$ & 100 & & 119.4 & & $0.030(0.003)$ & 0.006 & 4.3 & 0.03 \\
\hline$\ldots \ldots \ldots$ F5 & 100 & & 167.2 & & $0.024(0.003)$ & 0.005 & 4.0 & 0.03 \\
\hline$\ldots \ldots \ldots$ F6 & 100 & & 96.2 & & $0.024(0.003)$ & 0.008 & 2.6 & 0.03 \\
\hline UGC 8882 F1 & 100 & 25.34 & 222.4 & 958.3 & $0.076(0.004)$ & 0.003 & 19.8 & 0.00 \\
\hline$\ldots \ldots \ldots F \mathrm{~F} 2$ & 100 & & 139.7 & & $0.090(0.009)$ & 0.005 & 15.4 & 0.01 \\
\hline$\ldots \ldots \ldots$ F3 & 100 & & 141.7 & & $0.081(0.003)$ & 0.004 & 16.7 & 0.01 \\
\hline$\ldots \ldots \ldots \mathrm{F} 4$ & 100 & & 162.1 & & $0.084(0.003)$ & 0.005 & 14.3 & 0.01 \\
\hline$\ldots \ldots \ldots$ F5 & 100 & & 114.0 & & $0.090(0.002)$ & 0.006 & 13.1 & 0.01 \\
\hline$\ldots \ldots \ldots$ F6 & 100 & & 72.4 & & $0.085(0.003)$ & 0.007 & 10.7 & 0.01 \\
\hline$\ldots \ldots \ldots$ F7 & 100 & & 15.6 & & $0.125(0.010)$ & 0.019 & 6.3 & 0.01 \\
\hline$\ldots \ldots \ldots \mathrm{F} 8$ & 100 & & 17.5 & & $0.149(0.010)$ & 0.028 & 5.1 & 0.01 \\
\hline
\end{tabular}


Table 5. Fluctuation magnitudes and local colours for each SBF field in the dwarfs after correction for Galactic extinction.

\begin{tabular}{|c|c|c|c|}
\hline $\begin{array}{l}\text { Name } \\
\text { (1) }\end{array}$ & $\begin{array}{c}\mathrm{A}_{R} \\
(\mathrm{mag}) \\
(2)\end{array}$ & $\begin{array}{c}\bar{m}_{R}^{0} \\
(\mathrm{mag}) \\
(3)\end{array}$ & $\begin{array}{c}(B-R)_{0} \\
(\mathrm{mag}) \\
(4)\end{array}$ \\
\hline NGC 1703 F1 & $0.26 \pm 0.04$ & $26.89 \pm 0.089$ & $1.08 \pm 0.10$ \\
\hline .. F 2 & & $27.00 \pm 0.092$ & $1.14 \pm 0.12$ \\
\hline$\ldots \mathrm{F} 3$ & & $26.79 \pm 0.086$ & $1.08 \pm 0.11$ \\
\hline$\ldots \ldots \mathrm{F} 4$ & & $27.04 \pm 0.093$ & $1.14 \pm 0.12$ \\
\hline F5 & & $26.93 \pm 0.076$ & $1.10 \pm 0.09$ \\
\hline KDG 61 F1 & $0.19 \pm 0.03$ & $26.47 \pm 0.077$ & $1.09 \pm 0.12$ \\
\hline$\ldots \ldots \mathrm{F} 2$ & & $26.41 \pm 0.076$ & $1.11 \pm 0.13$ \\
\hline$\ldots \ldots$ F3 & & $26.46 \pm 0.149$ & $1.10 \pm 0.12$ \\
\hline$\ldots \ldots \mathrm{F} 4$ & & $26.40 \pm 0.125$ & $1.09 \pm 0.12$ \\
\hline$\ldots$ F5 & & $26.45 \pm 0.077$ & $1.10 \pm 0.11$ \\
\hline . F6 & & $26.36 \pm 0.091$ & $1.11 \pm 0.14$ \\
\hline UGCA 200 F1 & $0.13 \pm 0.02$ & $27.45 \pm 0.087$ & $1.24 \pm 0.14$ \\
\hline$\ldots \ldots \mathrm{F} 2$ & & $28.11 \pm 0.113$ & $1.31 \pm 0.10$ \\
\hline$\ldots \ldots \mathrm{F} 3$ & & $27.87 \pm 0.069$ & $1.28 \pm 0.09$ \\
\hline$\ldots \ldots \ldots \mathrm{F} 4$ & & $27.94 \pm 0.095$ & $1.31 \pm 0.09$ \\
\hline$\ldots \ldots$ F5 & & $27.80 \pm 0.097$ & $1.24 \pm 0.13$ \\
\hline $\begin{array}{ll}\text { UGC } 5442 \quad \text { F1 } \\
\end{array}$ & $0.14 \pm 0.02$ & $26.70 \pm 0.197$ & $1.24 \pm 0.08$ \\
\hline$\ldots F$ F2 & & $26.80 \pm 0.080$ & $1.22 \pm 0.08$ \\
\hline$\ldots \ldots \mathrm{F} 3$ & & $26.77 \pm 0.092$ & $1.24 \pm 0.08$ \\
\hline$\ldots \ldots \mathrm{F} 4$ & & $26.71 \pm 0.077$ & $1.22 \pm 0.08$ \\
\hline$\ldots \ldots \mathrm{F} 5$ & & $26.71 \pm 0.073$ & $1.23 \pm 0.10$ \\
\hline .....F6 & & $26.84 \pm 0.062$ & $1.23 \pm 0.09$ \\
\hline NGC 5944 F1 & $0.08 \pm 0.01$ & $29.02 \pm 0.131$ & $1.01 \pm 0.07$ \\
\hline$\ldots \mathrm{F} 2$ & & $28.96 \pm 0.092$ & $1.00 \pm 0.08$ \\
\hline$\ldots \mathrm{F} 3$ & & $29.03 \pm 0.093$ & $1.01 \pm 0.06$ \\
\hline$\ldots \ldots \mathrm{F} 4$ & & $28.87 \pm 0.070$ & $1.01 \pm 0.06$ \\
\hline$\ldots$ F5 & & $28.93 \pm 0.085$ & $1.00 \pm 0.09$ \\
\hline NGC 4150 F1 & $0.05 \pm 0.01$ & $29.54 \pm 0.063$ & $1.24 \pm 0.07$ \\
\hline$\ldots \ldots \ldots \ldots \mathrm{F} 2$ & & $29.64 \pm 0.054$ & $1.26 \pm 0.05$ \\
\hline$\ldots \mathrm{F} 3$ & & $29.35 \pm 0.084$ & $1.23 \pm 0.08$ \\
\hline$\ldots \mathrm{F} 4$ & & $29.48 \pm 0.079$ & $1.24 \pm 0.06$ \\
\hline$\ldots$ F5 & & $29.47 \pm 0.081$ & $1.25 \pm 0.05$ \\
\hline ...F6 & & $28.98 \pm 0.099$ & $1.17 \pm 0.13$ \\
\hline BTS $128 \mathrm{~F} 1$ & $0.07 \pm 0.01$ & $29.68 \pm 0.085$ & $1.23 \pm 0.06$ \\
\hline$\ldots \ldots \ldots \mathrm{F} 2$ & & $29.51 \pm 0.080$ & $1.24 \pm 0.05$ \\
\hline$\ldots \ldots \ldots \ldots \mathrm{F} 3$ & & $29.33 \pm 0.073$ & $1.21 \pm 0.10$ \\
\hline$\ldots \ldots \mathrm{F} 4$ & & $29.64 \pm 0.047$ & $1.23 \pm 0.07$ \\
\hline$\ldots \mathrm{F} 5$ & & $29.50 \pm 0.055$ & $1.21 \pm 0.08$ \\
\hline . F6 & & $29.64 \pm 0.065$ & $1.23 \pm 0.05$ \\
\hline UGC 7639 F1 & $0.03 \pm 0.00$ & $27.95 \pm 0.057$ & $0.90 \pm 0.04$ \\
\hline$\ldots \ldots \ldots \ldots \mathrm{F} 2$ & & $28.02 \pm 0.069$ & $0.96 \pm 0.06$ \\
\hline$\ldots \ldots \ldots \ldots \mathrm{F} 3$ & & $27.89 \pm 0.061$ & $0.93 \pm 0.05$ \\
\hline$\ldots \ldots \ldots \ldots \mathrm{F} 4$ & & $28.03 \pm 0.063$ & $0.95 \pm 0.06$ \\
\hline$\ldots \ldots \ldots \ldots \mathrm{F} 5$ & & $27.88 \pm 0.065$ & $0.95 \pm 0.05$ \\
\hline .....F6 & & $27.88 \pm 0.077$ & $0.97 \pm 0.07$ \\
\hline$\ldots \ldots \ldots$ F7 & & $28.05 \pm 0.058$ & $0.96 \pm 0.05$ \\
\hline$\ldots \ldots \ldots \ldots \mathrm{F} 8$ & & $27.98 \pm 0.069$ & $0.97 \pm 0.06$ \\
\hline UGC 8799 F1 & $0.07 \pm 0.01$ & $29.08 \pm 0.123$ & $1.24 \pm 0.05$ \\
\hline$\ldots \ldots \mathrm{F} 2$ & & $29.13 \pm 0.105$ & $1.24 \pm 0.05$ \\
\hline$\ldots \ldots \mathrm{F} 3$ & & $29.18 \pm 0.085$ & $1.25 \pm 0.05$ \\
\hline$\ldots \ldots \ldots \ldots \mathrm{F} 4$ & & $29.10 \pm 0.099$ & $1.23 \pm 0.08$ \\
\hline$\ldots \ldots$ F5 & & $29.31 \pm 0.122$ & $1.23 \pm 0.06$ \\
\hline$\ldots \mathrm{F} 6$ & & $29.35 \pm 0.147$ & $1.22 \pm 0.09$ \\
\hline UGC 8882 F1 & $0.02 \pm 0.00$ & $28.13 \pm 0.058$ & $1.22 \pm 0.05$ \\
\hline$\ldots \ldots \mathrm{F} 2$ & & $27.94 \pm 0.108$ & $1.21 \pm 0.06$ \\
\hline$\ldots \mathrm{F} 3$ & & $28.05 \pm 0.047$ & $1.21 \pm 0.06$ \\
\hline$\ldots \ldots \mathrm{F} 4$ & & $28.01 \pm 0.050$ & $1.21 \pm 0.06$ \\
\hline$\ldots \ldots \mathrm{F} 5$ & & $27.95 \pm 0.043$ & $1.20 \pm 0.07$ \\
\hline$\ldots \ldots \ldots \ldots$ F6 & & $28.01 \pm 0.055$ & $1.18 \pm 0.11$ \\
\hline$\ldots \ldots$ F7 & & $27.59 \pm 0.146$ & $1.07 \pm 0.21$ \\
\hline$\ldots \ldots \ldots \ldots$ F8 & & $27.39 \pm 0.125$ & $1.09 \pm 0.18$ \\
\hline
\end{tabular}

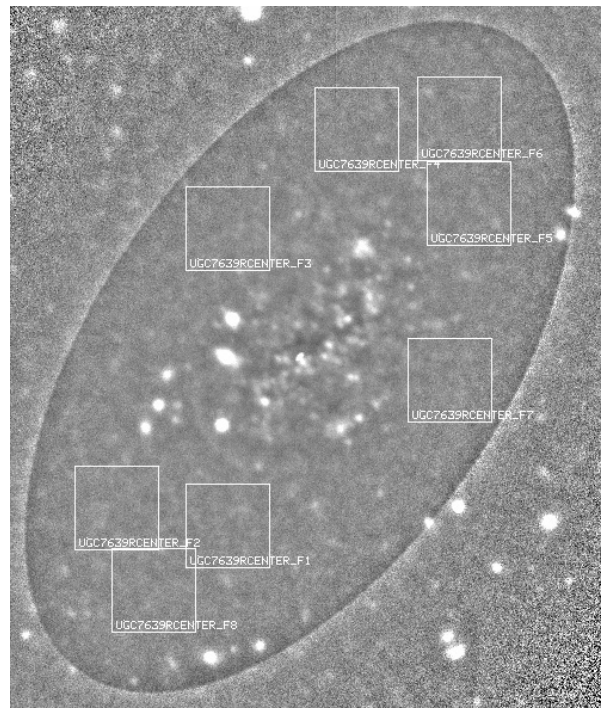

Fig. 10. Residual $R$-band image of UGC 7639 with the eight SBF fields indicated.

\section{UGC 8882}

Neither a velocity nor a distance was available for this nucleated dwarf elliptical galaxy. Bremnes et al. (1999) claim the galaxy is part of the M101 group and reported a mean $B-R$ colour of 1.29. The Cepheid based distance modulus for M101 is $(m-M)_{0}=29.28 \pm 0.14$ mag (Stetson et al. 1998 and references therein). We analysed eight independent SBF fields across the galaxy's surface (Fig. 13, left panels). Because of the observed linear relation between colour and SBF magnitude for the different fields we used the linear branch of the calibration diagram. The derived distance modulus is $(m-M)_{\mathrm{SBF}}=$ $29.60 \pm 0.20$ mag (see Fig. 13, right panel). This result is consistent with UGC 8882 being a member of the M101 group.

\section{Summary and conclusions}

We have analysed $B R$-band CCD images of nine nearby dwarf ellipticals and one S0 galaxy. We have measured their stellar $R$-band surface brightness fluctuation magnitudes $\bar{m}_{R}$ and $(B-R)_{0}$ colours in 61 galaxy fields. The resulting distances were compared with existing distances measured with the TRGB method and SBF method. Agreement between our distances and those of TRGB and previous SBF measurements was very high; all distances agreed within $0.1 \mathrm{mag}$.

Our SBF distances are given in Table 6 together with heliocentric radial velocities and radial velocities relative to the centre of mass of the Local Group of galaxies. The latter were calculated from heliocentric radial velocities following de Vaucouleurs et al. (1991) and using our distances to the galaxies. The Local Group centre of mass was assumed to lie exactly half way between the Milky Way and M31. The SBF distance to velocity relation positions BTS 128, UGC 7639 and UGC 8799 quite well on the Hubble flow. This is not the case with NGC 4150, KDG 61 and UGC 5442.

Our SBF distance to NGC 4150 of $14.4 \pm 0.7 \mathrm{Mpc}$ is in good agreement with earlier SBF distances (Tonry et al. 2001; Jensen et al. 2003) of $13.7 \pm 1.7 \mathrm{Mpc}$ and $12.8 \pm 1.5 \mathrm{Mpc}$, 

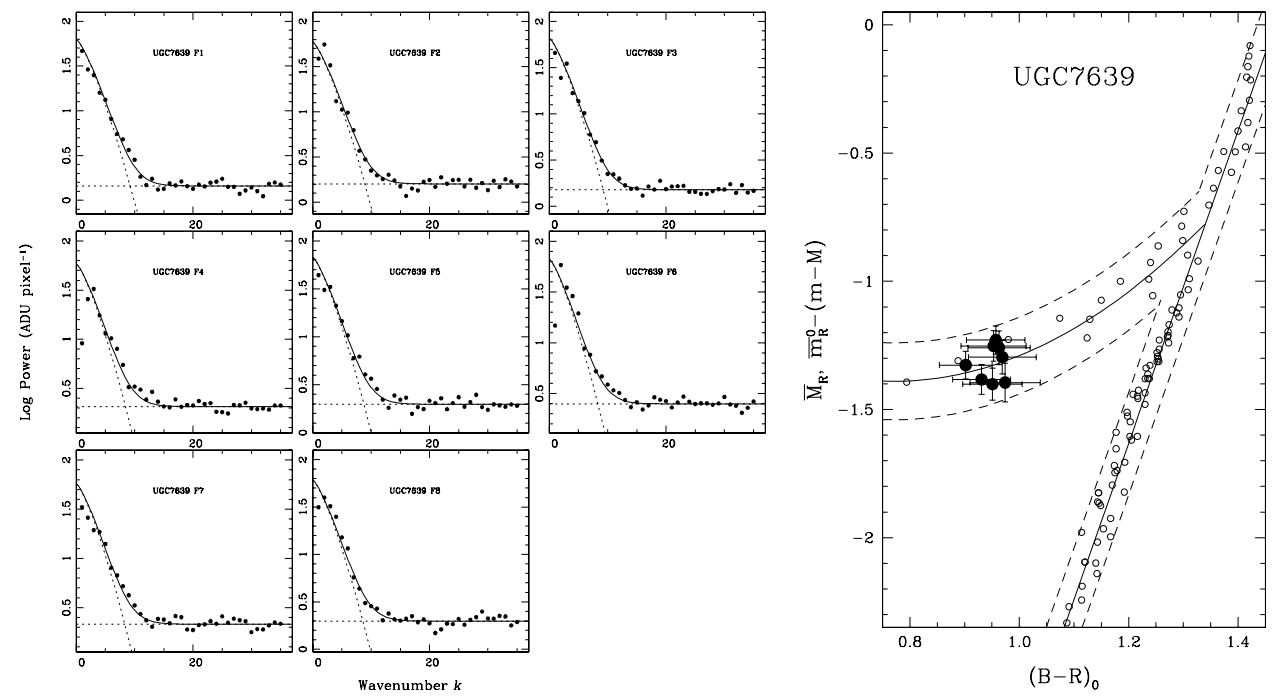

Fig. 11. Left: eight fields were selected for the SBF analysis in UGC 7639. The signal-to-noise in the power spectra is high. The power spectra of the SBF fields (filled circles) are well fitted by the sum (solid line) of a scaled version of the power spectrum of the PSF and a constant (dashed lines). The wavenumbers 1-4 were not considered for the fit. Right: eight fields were analysed to derive the distance of UGC 7639. A shift by 29.27 mag yields the best fit of the data to the calibration diagram.
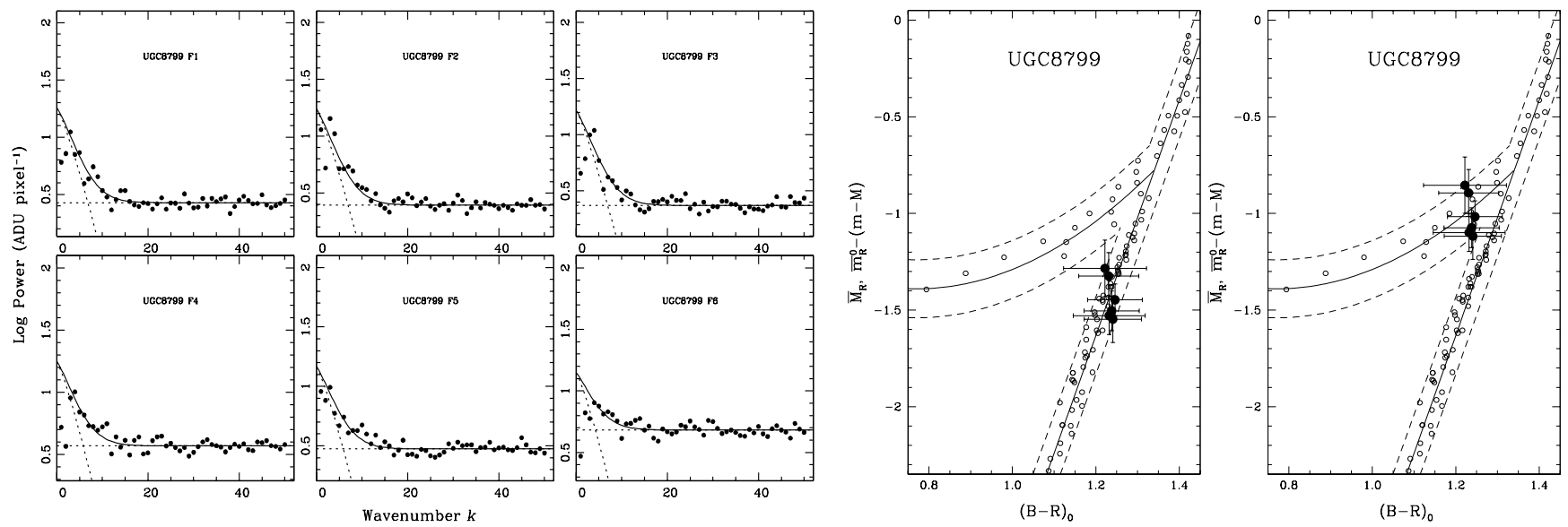

Fig. 12. Left: six fields were selected for the SBF analysis in UGC 8799. The signal-to-noise in the power spectra is not as high as measured in the other galaxies due to the larger distance of the galaxy at $\sim 13.2 \mathrm{Mpc}$. Nevertheless, the power spectra of the SBF fields (filled circles) are reasonably well fitted by the sum (solid line) of a scaled version of the power spectrum of the PSF and a constant (dashed lines). The wavenumbers 1-4 were not considered for the fit. Right: six fields were analysed to derive the distance of UGC 8799 . A shift by 30.19 mag or 30.61 mag yields the best fit of the data to the calibration diagram.

and places this galaxy almost $10 \mathrm{Mpc}$ behind the centre of Canes Venatici I cloud. All these estimates are consistent with Karachentsev et al. (2003) lower distance limit of 6.3 Mpc using the TRGB method in a study of CVn I cloud, and their very rough estimate of $20 \mathrm{Mpc}$, using the globular cluster luminosity function method. The galaxy might then be associated with the outskirts of the Virgo cluster. The velocity of NGC 4150 is rather low $\left(v_{\odot}=226 \mathrm{~km} \mathrm{~s}^{-1}\right)$, but it is difficult to say whether it is discordant with either our distance estimate of $14.4 \pm 0.7 \mathrm{Mpc}$ or Karachentsev et al.'s (2003) $\approx 20 \mathrm{Mpc}$. Solanes et al. (2002) have found that most Virgo galaxies in the region closest to us, corresponding to our distance to NGC 4150, have high radial velocities outward from the cluster centre (with similarly unusually high velocities away from us for Virgo cluster galaxies in the region behind the centre of the cluster). The angular separation of NGC 4150 from the cluster centre is 19 degrees. If it has a high outward velocity from the cluster centre, its line-of-sight velocity could be quite low. The observed velocity of NGC 4150 can be consistent with membership in the Virgo cluster, at its outer edge.

Our SBF distance to KDG 61 confirms the existing TRGB distance (Karachentsev et al. 2000) and thus the membership in M 81 group. Another M 81 group member is confirmed as our SBF distance to UGC 5442 agrees well with a previous TRGB distance (Karachentsev et al. 2000). Radial velocities of KDG 61 and UGC 5442 are reasonable relative to M 81 velocity of $v_{\odot}=-34 \pm 4$ (de Vaucouleurs et al. 1991) or $v_{\mathrm{LG}} \approx$ $96 \mathrm{~km} \mathrm{~s}^{-1}$.

UGC 7639 had only a tentative distance measured with brightest blue and red stars (Makarova et al. 1998) before our 

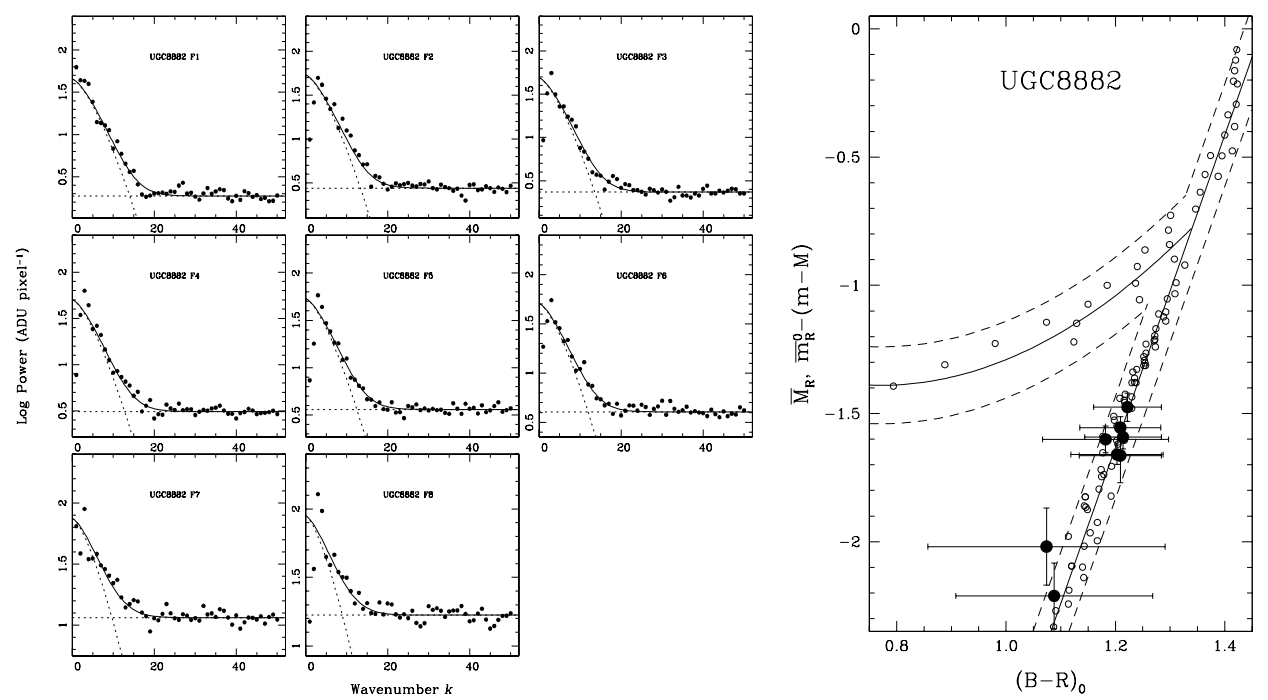

Fig. 13. Left: eight fields were selected for the SBF analysis in UGC 8882. The signal-to-noise in the power spectra is not as high as measured in the other galaxies due to the larger distance of the galaxy at $14.4 \mathrm{Mpc}$. Nevertheless, the power spectra of the SBF fields (filled circles) are well fitted by the sum (solid line) of a scaled version of the power spectrum of the PSF and a constant (dashed lines). The wavenumbers $1-4$ were not considered for the fit. Right: eight fields were analysed to derive the distance of UGC 8882 . A shift by 29.60 mag yields the best fit of the data to the calibration diagram.

Table 6. SBF distances of the sample galaxies with the ambiguous case listed twice. Heliocentric and Local Group barycentric velocities are also listed if available.

\begin{tabular}{|c|c|c|c|c|}
\hline $\begin{array}{l}\text { Galaxy } \\
\text { Name } \\
\text { (1) }\end{array}$ & $\begin{array}{c}(m-M)_{0} \\
(\mathrm{mag}) \\
(2)\end{array}$ & $\begin{array}{c}D \\
(\mathrm{Mpc}) \\
(3)\end{array}$ & $\begin{array}{c}v_{\odot} \\
\left(\mathrm{km} \mathrm{s}^{-1}\right) \\
(4)\end{array}$ & $\begin{array}{c}v_{\mathrm{LG}} \\
\left(\mathrm{km} \mathrm{s}^{-1}\right) \\
(5)\end{array}$ \\
\hline UGC 1703 & $28.11 \pm 0.15$ & $4.2 \pm 0.3$ & - & - \\
\hline KDG 61 & $27.61 \pm 0.17$ & $3.3 \pm 0.3$ & -135 & -4 \\
\hline UGCA 200 & $29.01 \pm 0.27$ & $6.3 \pm 0.8$ & - & - \\
\hline UGC 5442 & $27.74 \pm 0.18$ & $3.5 \pm 0.3$ & -18 & 106 \\
\hline UGC 5944 & $30.22 \pm 0.17$ & $11.1 \pm 0.9$ & - & - \\
\hline NGC 4150 & $30.79 \pm 0.11$ & $14.4 \pm 0.7$ & 226 & 207 \\
\hline BTS 128 & $31.02 \pm 0.25$ & $16.0 \pm 1.9$ & 1139 & 1129 \\
\hline UGC 7639 & $29.27 \pm 0.16$ & $7.1 \pm 0.6$ & 382 & 447 \\
\hline UGC 8799 & $30.19 \pm 0.23$ & $10.9 \pm 1.2$ & 1132 & 1094 \\
\hline- & $30.61 \pm 0.26$ & $13.2 \pm 1.7$ & & \\
\hline UGC 8882 & $29.60 \pm 0.20$ & $8.3 \pm 0.8$ & - & - \\
\hline
\end{tabular}

SBF distance, which is in relatively good agreement with the earlier distance and confirms the location of this galaxy in the Canes Venatici II cloud.

Distances to galaxies UGC 1703, UGCA 200, UGC 5944 and UGC 8882 were not previously known. We have provided accurate SBF distances to these galaxies. Our distances suggest UGC 1703 may be a distant companion of NGC 784, UGCA 200 may not be a companion of NGC 3115 as was assumed before, UGC 5944 is most certainly a member of Leo I group and UGC 8882 seems to be a member of M 101 group. Likewise only radial velocities were known for galaxies BTS 128 and UGC 8799 before our SBF distances, which confirm the membership of BTS 128 in Coma I group and suggest UGC 8799 lies at the outskirts of Virgo I cluster. Its angular separation from the cluster centre, at M 87 location of $12^{\mathrm{h}} 31^{\mathrm{m}}$ in RA and $+12^{\circ} 23^{\mathrm{m}}$ in Dec $(\mathrm{J} 2000.0)$, is 21 degrees.
The SBF distances we have presented continue to support the understanding of the distribution of dwarf galaxies in galaxy groups and intermediate space in the Local Group neighbourhood. They also demonstrate well the feasibility of the Surface Brightness Fluctuation method in determining accurate distances with $2 \mathrm{~m}$ class ground-based telescopes out to the near side of the Virgo cluster.

Acknowledgements. The Nordic Optical Telescope is operated on the island of La Palma jointly by Denmark, Finland, Iceland, Norway, and Sweden, in the Spanish Observatorio del Roque de los Muchachos of the Instituto de Astrofísica de Canarias. We thank Kari Nilsson for his help with the observations and the Academy of Finland for support through its funding of the ANTARES programme. Financial support for RR has been provided by Finnish Graduate School in Space Physics and Astronomy and by the Academy of Finland through funding of the project "Calculation of Orbits". This research has made use of the NASA/IPAC Extragalactic Database (NED), which is operated by the Jet Propulsion Laboratory, California Institute of Technology, under contract with the National Aeronautics and Space Administration. We are grateful to the referee Enzo Brocato for his useful comments.

\section{References}

Baryshev, Yu. V., Chernin, A. D., \& Teerikorpi, P. 2001, A\&A, 378, 729

Binggeli, B., Tarenghi, M., \& Sandage, A. 1990, A\&A, 228, 42

Bremnes, T., Binggeli, B., \& Prugniel, P. 1999, A\&AS, 137, 337

Côté, S., Freeman, K. C., Carignan, C., \& Quinn, P. J. 1997, AJ, 114, 1313

Cowie, L. L., Gardner, J. P., Hu, E. M., et al. 1994, ApJ, 434, 114

de Jong, R. S. 1996, A\&A, 313, 377

de Vaucouleurs, G., de Vaucouleurs, A., Corwin, H. G., et al. 1991,

Third Reference Catalogue of Bright Galaxies Vol. I (New York:

Springer-Verlag), 55

Drozdovsky, I. O., \& Karachentsev, I. D. 2000, A\&AS, 142, 425 
Ferguson, H., \& Sandage, A. 1990, AJ, 100, 1

Fisher, H., Huchra, J. P., Strauss, M. A., et al. 1995, ApJS, 100, 69 Geller, M. J., Kurtz, M. J., Wegner, G., et al. 1997, AJ, 114, 2205 Jensen, J. B., Tonry, J. L., \& Luppino, G. A. 1998, ApJ, 505, 111 Jensen, J. B., Tonry, J. L., Barris, B. J., et al. 2003, ApJ, 583, 712 Jerjen, H. 2003, A\&A, 398, 63

Jerjen, H., Freeman, K. C., \& Binggeli, B. 1998, AJ, 116, 2873

Jerjen, H., Binggeli, B., \& Freeman, K. C. 2000a, AJ, 119, 593

Jerjen, H., Freeman, K. C., \& Binggeli, B. 2000b, AJ, 119, 166

Jerjen, H., Rekola, R., Takalo, L., Coleman, M., \& Valtonen, M. 2001, A\&A, 380, 90

Jerjen, H., Binggeli, B., \& Barazza, F. D. 2004, AJ, 127, 771

Johnson, R. A., Lawrence, A., Terlevich, R., \& Carter, D. 1997, MNRAS, 287, 333

Karachentseva, V. E., \& Karachentsev, I. D. 1998, A\&AS, 127, 409

Karachentsev, I. D., Karachentseva, V. E., Dolphin, A. E., et al. 2000, A\&A, 363, 117

Karachentsev, I. D., Sharina, M. E., Dolphin, A. E., et al. 2003, A\&A, 398, 467

Landolt, A. U. 1992, AJ, 104, 340
Makarova, L., Karachentsev, I., Takalo, L. O., Heinämäki, P., \& Valtonen, M. 1998, A\&AS, 128, 459

Miller, B. W., Lotz, J. M., Ferguson, H. C., Stiavelli, M., \& Whitmore, B. C. 1998 , ApJL, 508, 133

Moore, B., Ghigna, S., Governato, F., et al. 1999, ApJL, 524, 19

Parodi, B. R., Barazza, F. D., \& Binggeli, B. 2002, A\&A, 388, 29

Sandage, A., \& Bedke, J. 1994, The Carnegie atlas of galaxies (Washington, DC: Carnegie Institution)

Sandage, A., \& Binggeli, B. 1984, AJ, 89, 919

Schlegel, D. J., Finkbeiner, D. P., \& Davis, M. 1998, ApJ, 500, 525

Silbermann, N. A., Harding, P., Madore, B. F., et al. 1996, ApJ, 470, 1 Simien, F., \& Prugniel, Ph. 2002, A\&A, 384, 371

Solanes, J. M., Sanchis, T., Salvador-Solé, E., Giovanelli, R., \& Haynes, M. P. 2002, AJ, 124, 2440

Tanaka, K. I. 1985, PASJ, 37, 133

Tonry, J. L., \& Schneider, D. P. 1988, AJ, 96, 807

Tonry, J. L., Dressler, A., Blakeslee, J. P., et al. 2001, ApJ, 546, 681

Trentham, N., \& Tully, R. B. 2002, MNRAS, 335, 712

Wegner, G., Thorstensen, J. R., Kurtz, M. J., et al. 2001, AJ, 122, 2893 